Spin-up of homogeneous and stratified fluids

Duck, P. and Foster, M.R.

2001

MIMS EPrint: 2006.252

Manchester Institute for Mathematical Sciences

School of Mathematics

The University of Manchester

\footnotetext{
Reports available from: http://eprints.maths.manchester.ac.uk/

And by contacting: The MIMS Secretary

School of Mathematics

The University of Manchester

Manchester, M13 9PL, UK
} 


\title{
SPIN-UP OF HoMOgeneous AND STRATIFied Fluids
}

\author{
PW Duck \\ Department of Mathematics, University of Manchester, Manchester, M13 9PL, \\ United Kingdom; e-mail: duck@ma.man.ac.uk \\ MR Foster \\ Department of Aerospace Engineering, Applied Mechanics and Aviation, The Ohio State \\ University, Columbus, Ohio 43210; e-mail: foster@r1tel.eng.ohio-state.edu
}

Key Words rotating flows, spin-down, spin-over, conical flows

- Abstract We consider the manner in which a container filled with viscous fluid adjusts to changes in its rotation rate. We begin with homogeneous flows involving small departures in rotation rate from an initial state of solid-body rotation in an axisymmetric container. This is followed by a summary of other more recent developments, including weakly and fully nonlinear calculations and comparison with experiment and the question of spin-down. The question of "spin-over" is addressed, followed by a brief synopsis of free-surface effects, and a discussion of nonaxisymmetric spin-up. The second part of the review focuses on the effects of stratification on the spin-up process. Linearized (low Rossby number) spin-up within a cylindrical container is described. Thereafter, both experimental and nonlinear computational results are described and compared. The final section focuses on stratified spin-up and spin-down in conical geometries, and a number of comparisons between theory and experiment are given.

\section{INTRODUCTION}

A closed container filled with fluid (of kinematic viscosity coefficient $v$ ) is rotating rigidly about some axis (at an angular speed $\Omega$ ), and has been in that state long enough so that the fluid inside is rotating rigidly with the container. The fluid inside may be homogeneous, or electrically conducting, or density stratified. At $t=0$, the rotation rate of the container is suddenly altered by an amount $\Delta \Omega$, and the fluid inside adjusts in time to a new rotational state. The process whereby this adjustment occurs has come to be known as spin-up $(\Delta \Omega>0)$ or spin-down $(\Delta \Omega$ $<0$ ); a natural and key parameter to define in flows of this type is therefore the Rossby number, $\epsilon \equiv \Delta \Omega / \Omega$, a measure of the relative change in rotation rate. A further important nondimensional parameter is the Ekman number of the flow 
$E \equiv v / \Omega h^{2}$, where $h$ is a vertical length scale associated with the container. Most attention over the years has focused on the small-Ekman-number limit, this being the regime of most practical and theoretical interest. Twenty-five years ago, Benton \& Clark (1974) provided a thorough summary of work on spin-up to that point in time. There is no need here to repeat their summary in detail, though it is important to reiterate results from some of the most important papers to which they refer, in order to place the work published since that time in a proper context.

The modern era of spin-up studies was initiated by the landmark paper by Greenspan \& Howard (1963), who considered the effect of small changes of rotation rate of a container on flow (i.e. the small Rossby number limit). The smallness of $\epsilon$ permits a linearization about the original uniform state; in particular these authors were able to highlight the importance of the spin-up timescale, $h /(\nu \Omega)^{1 / 2}$.

In subsequent studies a proper understanding of a number of related spin-up problems has emerged. It turns out that the overall process involves elucidation of the differing dynamics that occur on various timescales of the problem, which for homogeneous fluids are $\Omega^{-1}, h /(v \Omega)^{1 / 2}$, and $h^{2} / \nu$, the latter of which is a (long) timescale for diffusion; for small Ekman number, $E$, these scales are distinct. It is precisely this scale separation that makes these problems tractable by means of asymptotic methods. If the fluid is stratified, there are two additional timescales, $N^{-1}$ and $h^{2} / \kappa$, where $N$ is the Brunt-Väisälä frequency associated with the (stable) stratification, and $\kappa$ is the diffusion coefficient for the density anomaly; electrical conduction effects introduce yet more timescales. Various researchers recognized early on that there are other significant effects due to differing geometrical shapes of the container; in particular, whether the container is axisymmetric is crucial to the character of the spin-up processes.

A feature of these spin-up problems and in the concomitant development of steady, rapidly rotating flows - in which the key paper is by Stewartson (1957) is the active role of the boundary layers in the flow evolution. Though it was commonly understood that boundary motion could induce significant core flowsas in the case of the Bödewadt problem, for example- the synergistic interaction of an inviscid core with the wall layers to accomplish spin-up on a timescale that cannot be deduced solely by either inviscid or diffusive arguments is at first a surprising feature of these flows, and its description at that time was a real triumph of matched-asymptotic-expansion methods.

While some effort since 1974 has continued to utilize asymptotic methods to examine low Rossby number limit cases in detail, many new and interesting experiments have been reported, and, not surprisingly, there has also been a substantial effort in numerical modeling of such flows.

In this article, we first briefly reprise some of the early work in linearized (small Rossby number) homogeneous spin-up. This summary is followed by a discussion on finite-amplitude effects in spin-up, and then we detail some of the literature on spin-up in partially filled containers, which inevitably involve free-surface effects. There follows a discussion of spin-down (which in the nonlinear regime is 
of course quite different from spin-up), and then we consider the case of spin-over (when the final rotation is in the opposite sense to the initial rotation). To conclude our treatment on homogeneous fluids, we consider recent developments for spin-up in nonaxisymmetric containers. We then move on to describe a number of results for stratified spin-up within cylinder-like containers, considering first the small Rossby number limit, but then extending our discussion to finite Rossby number flows. Finally, we turn to recent developments with regard to spin-up and spin-down within a container with a conical bottom (again including the effects of stratification).

In a short review such as this, it is impossible to include references to all aspects of the spin-up literature, in all its variety. In particular we specifically exclude from our discussion electromagnetic effects in spin-up, partly because some attention was already paid to it in Benton's \& Clark's (1974) review. Interested readers can refer to a series of papers on the subject, including a number of relatively early papers by Benton \& Loper (1969), Loper \& Benton (1970), Loper (1971), Chawla (1972), and Benton (1973), and more recent work on the subject by Berman et al (1978) and Ungarish (1997). We also exclude from our survey the important area of the spin-up in a mixture of two fluids (see, for example, Amberg \& Ungarish 1993). Finally, the considerable literature on unsteady processes in gas centrifuges (Hyun \& Park 1992, Lindblad et al 1994, among others) is also specifically excluded from our discussion.

Before we begin, a word about notation. Throughout this article, $(u, v, w)$ denote the fluid velocity components in the radial, azimuthal, and vertical directions, respectively. In addition, an asterisk superscript, ( $)^{*}$, will always denote dimensional quantities, with unstarred quantities being dimensionless. A prime affixed to any variable, ()$^{\prime}$, refers to quantities in an inertial frame of reference, and unprimed quantities are with respect to a frame rotating at angular velocity $\Omega$. Any deviations from these conventions are noted.

\section{HOMOGENEOUS FLUIDS}

The literature on homogeneous fluids is enormous, having grown substantially since the earlier review of Benton \& Clark (1974), who performed an admirable job in summarizing the early work.

\subsection{Linear, Axisymmetric Spin-Up}

As we have noted above, the seminal paper for small Rossby number spin-up is that of Greenspan \& Howard (1963). We reiterate here a few of the more important mathematical details of that work because it provides the framework for later parts of this review.

Consider a circular cylinder filled with fluid of viscosity $v$, rotating rigidly about its symmetry axis at angular velocity $\Omega$. At $t=0$, the cylinder is suddenly 
spun up to a larger (or smaller) angular velocity $\Omega(1+\epsilon)$. After a few revolutions, the thin Ekman layers on the end walls, in which viscous effects are confined, are established as essentially steady [see the more complete discussion of this point by Benton \& Clark (1974)]. On a somewhat longer timescale (longer than $\Omega^{-1}$ ), in the fluid core away from the boundaries, the swirl velocity component $v^{*}$ is of order $\Omega h$, whereas the radial and vertical components, $u^{*}$ and $w^{*}$, are much smaller than this. The resulting approximate momentum equations indicate that $v^{*}$ is independent of $z^{*}$, which eventually implies that

$$
\frac{\partial^{2} w^{*}}{\partial z^{* 2}}=0
$$

and for $\epsilon=o(1)$ and $E$ small, the azimuthal momentum equation becomes

$$
\frac{\partial v^{*}}{\partial t^{*}}+\frac{u^{*}}{r^{*}} \frac{\partial v^{*}}{\partial r^{*}}+2 \Omega u^{*}=0
$$

It is well known (see Greenspan 1968) that an Ekman layer on either a top or bottom bounding wall imposes a velocity compatibility condition on the geostrophic flow adjacent to the layer. For all $\epsilon \ll 1$, the axisymmetric version of that condition is

$$
w^{*}=\mp \frac{1}{2 r^{*}}\left(\frac{v}{\Omega}\right)^{\frac{1}{2}}\left[\frac{\partial\left(r^{*} v^{*}\right)}{\partial r^{*}}-\frac{\partial\left(r^{*} v_{b}^{*}\right)}{\partial r^{*}}\right] \text { on } z^{*}=\left\{\begin{array}{l}
h \\
0
\end{array}\right\},
$$

and $v_{b}^{*}=\epsilon \Omega r^{*}$ denotes the swirl velocity of the boundary. Recalling from Equation 1 that $w^{*}$ must vary linearly between the values at $z^{*}=0$ and $z^{*}=h$, the continuity equation then allows the radial velocity to be related to $v^{*}$,

$$
u^{*}=\frac{1}{h}\left(\frac{v}{\Omega}\right)^{\frac{1}{2}}\left(v^{*}-v_{b}^{*}\right)
$$

Substitution of this $u^{*}$ into Equation 2 gives the nonlinear equation for the swirl,

$$
\frac{\partial \tilde{v}^{*}}{\partial t^{*}}+\frac{2(\nu \Omega)^{\frac{1}{2}}}{h}\left(\tilde{v}^{*}-\tilde{v}_{b}^{*}\right)\left[1+\frac{\epsilon}{2 \Omega r^{*}} \frac{\partial\left(r^{*} \tilde{v}^{*}\right)}{\partial r^{*}}\right]=0,
$$

where $v^{*}=\epsilon \tilde{v}$. From this equation, the linearized solution (apparently for $\epsilon \ll 1$ ) is easily shown to be

$$
v^{*}=\epsilon \Omega r^{*}(1-\mathcal{E}), \quad \mathcal{E} \equiv \exp \left(-2(\nu \Omega)^{1 / 2} t^{*} / h\right) .
$$

Of perhaps more interest is the meridional circulation given by

$$
u^{*}=-\frac{\epsilon r^{*}(\nu \Omega)^{\frac{1}{2}}}{h} \mathcal{E}, \quad w^{*}=\epsilon(\nu \Omega)^{\frac{1}{2}}\left(\frac{1}{2}-\frac{z}{h}\right) \mathcal{E} .
$$

The order of these components confirms the initial hypothesis that the $u^{*}$ and $w^{*}$ are $o\left(v^{*}\right)$ if $E \ll 1$. 
On timescales of order $\Omega^{-1}$, inertial oscillations exist in the container (see Greenspan 1968), but they have small amplitude and decay over a viscous timescale; they appear not to be dynamically important to the spin-up mechanism. Agreement of this quasi-linear theory and experiment, without the waves included, has been shown consistently to be excellent (see Warn-Varnas et al 1978, for example). However, a recent interesting paper by Kerswell (1999) indicates that nonlinear effects (above a threshold amplitude) can lead to significant, large-scale effects from the waves.

Fluid carried toward the outer wall in the Ekman layers on the top and bottom of the container flows into the corners at $r^{*}=a$, and then into the boundary layers on the vertical side walls— the "1/4" and "1/3" Stewartson layers (Stewartson 1957). The fluid then flows radially out of these layers at $r^{*}=a-$, thus delivering the radial inflow required by solutions (Equation 7). That radial inflow in the interior turns upward or downward (according to Equation 7), and flows into the Ekman layers on the horizontal walls, thus completing the circulation. Hence, the spin-up is accomplished by high-momentum fluid being carried inward into the interior by this meridional-plane circulation. The meridional flow is shown schematically as Figure 1.

Finally, note that the related problem of the effect on the fluid (infinite in extent) above a rotating disk, whose angular velocity is changed abruptly (by a small amount), has been studied by Chawla (1976).

\subsection{Nonlinear, Axisymmetric Spin-Up}

A number of investigators have attempted to extend the solutions of Equation 5 into the nonlinear regime, beginning with Wedemeyer (1964). An extensive discussion of the contributions to date for the nonlinear analysis is given in Benton \& Clark (1974). It turns out that the solution given as Equation 6 is actually valid only for $\epsilon \ll E^{1 / 4}$. For values of $\epsilon$ of order $E^{1 / 4}$ or larger, but still small compared to one, all of the orderings go through and the solution to Equation 5 is

$$
\begin{aligned}
\tilde{v}^{*} & =\Omega r^{*} \frac{1-\mathcal{E}}{1+\epsilon \mathcal{E}}, \quad \text { for } r^{*}<r_{0}^{*}\left(t^{*}\right), \\
\tilde{v}^{*} & =\Omega r \frac{\epsilon+\mathcal{E}}{\epsilon(1-\mathcal{E})}-\frac{\Omega a^{2}}{r^{*}} \frac{1+\epsilon}{\epsilon(1-\mathcal{E})} \mathcal{E}, \quad \text { for } r_{0}^{*}\left(t^{*}\right)<r^{*}<a, \\
r_{0}^{*}\left(t^{*}\right) & =a\left(\frac{1+\epsilon \mathcal{E}}{1+\epsilon}\right)^{\frac{1}{2}} .
\end{aligned}
$$

This solution in essentially this form was given by Weidman (1976a); noteworthy is the front that propagates from the outer wall inward, terminating for long time at $a / \sqrt{1+\epsilon}$. Venezian (1970) showed that in this parameter regime, there is a shear layer of width $E^{1 / 4}$ at $r^{*}=r_{o}^{*}$.

Since the weakly nonlinear results were proposed, Greenspan \& Weinbaum (1965), Benton (1973), Weidman (1976a), and many others (see Benton \& Clark 


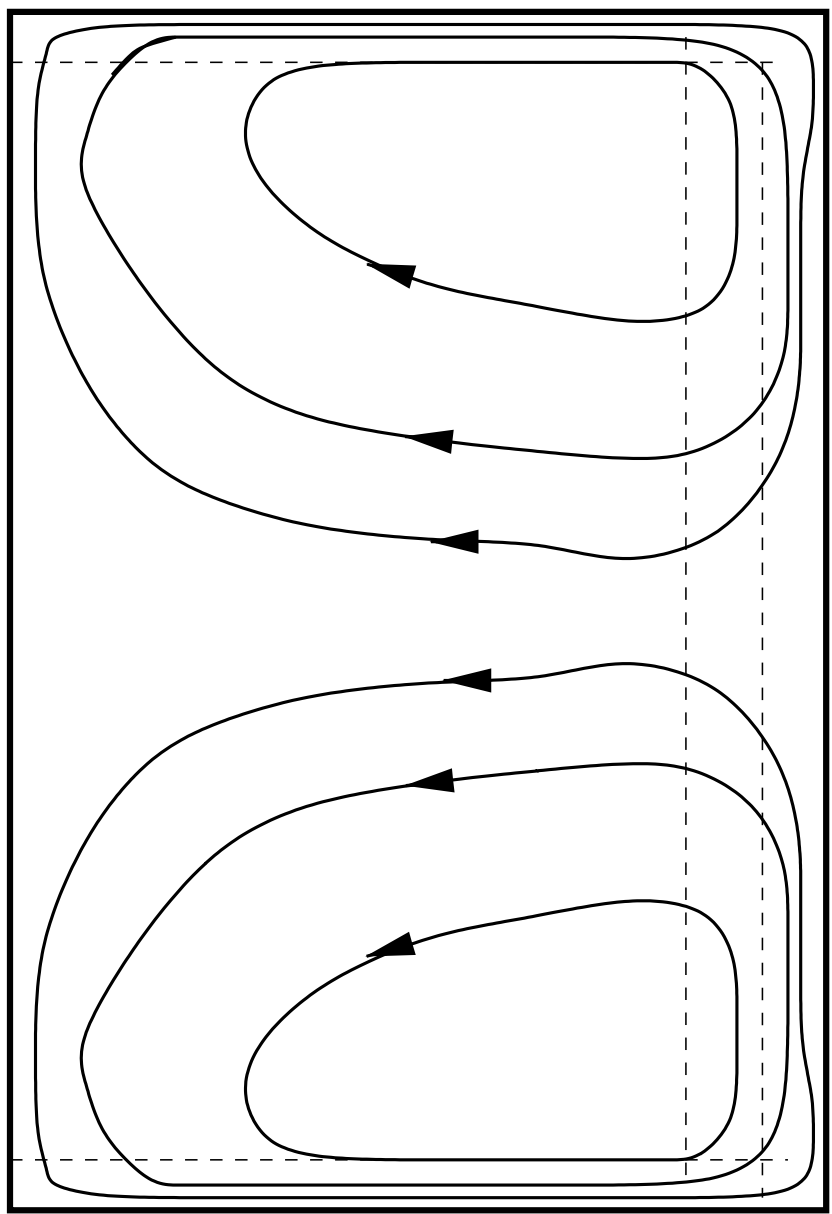

Figure 1 Schematic of meridional-plane streamlines for homogenous spin-up. The lefthand boundary is the container centerline. The dashed lines indicate Ekman layers and sidewall Stewartson layers, all of which transport mass.

1974) have sought to extend the validity of Equation 5 to include $\epsilon=O(1)$ because the assumptions leading to Equation 5 do not require that $\epsilon$ be small. In order to proceed, one must replace Equation 3 with a nonlinear version of the Ekman suction law. Typically, such a new entrainment law, with continuity, leads directly to a generalization of Equation 4, in a form

$$
u^{*}=E^{1 / 2} \mathcal{F}\left(v^{*}-v_{b}^{*}\right),
$$

for some function $\mathcal{F}$. Benton \& Clark (1974) present an extensive discussion of the determination of $\mathcal{F}$, which we do not repeat here. As an example, Figure 2, reproduced from Weidman (1976b), is a comparison of a spin-up experiment with 

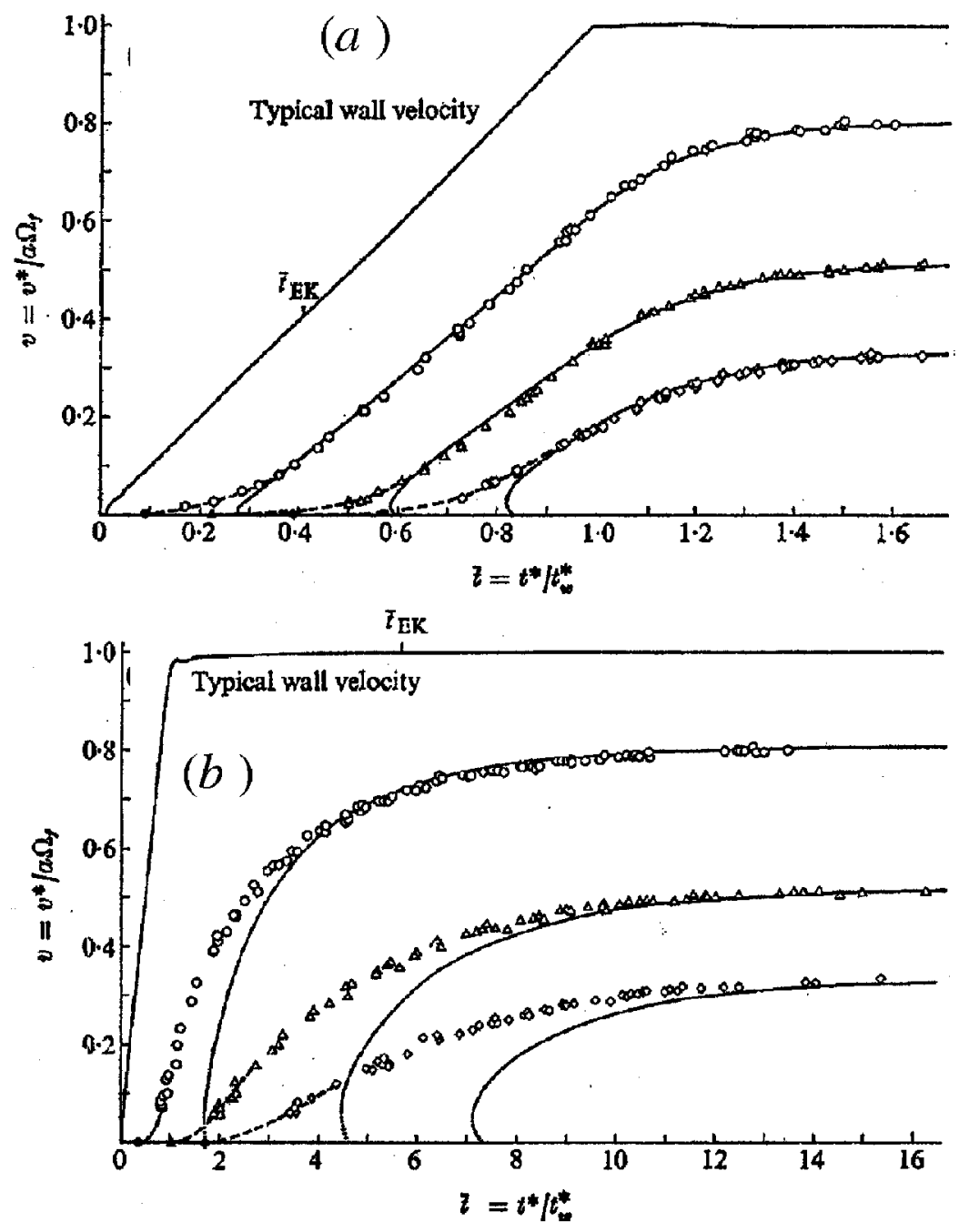

Figure 2 Spin-up from rest under constant angular acceleration, $\alpha ; t_{w} \equiv \Delta \Omega / \alpha . \mathrm{o}, r^{*} / a=$ $0.807 ; \triangle, 0.517 ; \diamond, 0.334$; 一, theoretical result. (a) $E=1.16 \times 10^{-7}, \sqrt{\alpha} / \Omega=0.00429$; (b) $E=9.91 \times 10^{-8}, \sqrt{\alpha} / \Omega=0.0113$. Filled symbols denote front location. Reproduced from Weidman (1976b).

a prediction based on such a model, for spin-up by means of a constant acceleration from rest. The theoretical results utilize a seventh-degree polynomial for $\mathcal{F}$, with coefficients chosen to agree with the computational results of Rogers \& Lance (1960). As Weidman notes in his paper, the agreement is best away from the front, and also at long times. 
There have been a number of concerns raised about the (Equation 9) ansatz for $\mathcal{F}$. Bennetts \& Hocking (1973) made a systematic attempt to generate such a nonlinear suction law, and concluded instead that it may not be viable. Watkins \& Hussey (1973) detail a number of difficulties, based on both experimental and numerical evidence, in particular noting that significant discrepancies can occur near the velocity front or when $\left(\frac{h}{a}\right)^{2} E^{\frac{1}{2}}(r(1-r))$ exceeds the value 0.20 . Both papers justifiably call into question the validity of Equation 9. On the other hand, there is no question (see Figure 1.4 in Greenspan 1968) that the inward-propagating front, which occurs in the results obtained using an amalgam of Equations 2 and 9 , is clearly observed in experiments for $\epsilon=O(1)$.

The former critiques have been given clearer elucidation in the recent paper by Hart (2000), who has used the software package MAPLE to obtain five terms in an $\epsilon$ asymptotic series for the Ekman suction. He notes that for $\epsilon=0.75$, for example, Ekman layer edge velocities other than the linear one studied by Rogers \& Lance (1960) give dramatically different suction laws. More troubling, however, is his point—also made in a different way by Bennetts \& Hocking (1973)—that the real difficulty is that in the nonlinear regime the Ekman suction is inherently nonlocal: Higher-order terms in the $\epsilon$ series for suction are functions of higherorder radial derivatives of $v^{*}$. Therefore, any suggestion that the suction velocity depends solely on the local vorticity is doomed at the outset-even for moderate values of $\epsilon$. In regions of high radial shear-near the outer wall and near the frontthe large, high-order radial derivatives in higher-order terms in the suction series become important, thus explaining the observed discrepancies near the front. One can show from Hart's series that all terms in his series are equally important as $\epsilon$ approaches $O(1)$.

Clearly, the presence of a front is not an artifact of Equation 4 but will exist for many choices of $\mathcal{F}$, because a solution qualitatively similar to Equation 8 can still be constructed. Indeed, as $\bar{t} \rightarrow \infty$, regardless of the validity of Equation 3 at earlier times, $v^{*} \rightarrow v_{b}^{*}$, and so the linear law, Equation 4 , does eventually become valid. One can show that the front is in general located at $r^{*}=c_{1}-c_{2} \exp (-\bar{t})$, but the constants $c_{1}$ and $c_{2}$ cannot be simply related to the initial conditions; recall that Weidman (1976b) notes that the best agreement between experiment and theory occurs at long spin-up times.

Thus, when $\epsilon$ is truly order one-as for the case of spin-up from rest-the foregoing theories lose validity and one must turn to computations. Since the review of Benton \& Clark (1974), many high-resolution numerical solutions to problems of spin-up and spin-down, for $\epsilon=O(1)$, have been published. An early attempt, utilizing a reduced set of equations [based on a linear relation between the radial and azimuthal velocity components, suggested by Wedemeyer (1964)], was made by Watkins \& Hussey (1977), who were able to compare their results, quite favorably, with previous theories. An important early contribution, involving the solution of the full governing equations, was that of Warn-Varnas et al (1978), who performed a careful comparison between their numerical results with both their own experiments and the asymptotic theories discussed above. 
From their reports of truncation error in their calculations, the Ekman layers appear to be well resolved. Most results presented are for $\epsilon=0.111$ and $E=$ $3.3 \times 10^{-3}$ to correspond with the experiments. This is an interesting choice because $\epsilon / E^{1 / 4}=0.461$, which is right in the crucial range for the validity of the Wedemeyer theory. Their observations of the swirl velocity component agree quite well with the numerical solutions and include a good representation of the oscillatory component of the flow. These inertial oscillations have small amplitude and are superposed on the slow decay, as Greenspan \& Howard (1963) predicted.

Subsequent to that work, Kitchens (1980) extended the range of Ekman numbers considered numerically, down to $2 \times 10^{-5}$, whereas Hyun et al (1983) presented numerical results down to $9.18 \times 10^{-6}$, and carried out comparisons with experiment [we note also that these authors had some criticism of the work of Wedemeyer (1964)]. More recent work was published by Ibrani \& Dwer (1987), in which the spin-up processes in both cylindrical and spherical geometries are reported; the aforementioned inertial oscillations were again much in evidence in this work.

\subsection{Nonlinear, Axisymmetric Spin-Down}

It has been long understood that spin-down is significantly different from spin-up in the nonlinear regime. Weidman (1976b) noted that centrifugal instabilities arise at the outer wall for spin-down and gradual deceleration (rather than impulsive spin-down) shifts the critical Reynolds number for instability to larger values. Later, computations and experiments by Neitzel \& Davis (1981) and Mathis \& Neitzel (1985) verified that such centrifugal instabilities arise (originating at the mid-plane), leading to the formation of an array of Taylor-Görtler vortices along the side wall. Figure 3, reproduced from Neitzel \& Davis (1981), shows these Taylor-Görtler vortices for impulsive spin-down to rest in a container with aspect ratio one. Note that as the spin-down proceeds, smaller vortices coalesce into larger ones. The enhanced mixing due to these Görtler vortices generates much more rapid spin-down than that predicted according to weakly nonlinear arguments (Neitzel \& Davis 1981).

The most recent treatments of this problem, with the best resolution, are by Lopez (1996) and Lopez \& Weidman (1996). They found that in addition to the centrifugal instabilities near the side walls, inflectionally unstable circular waves arise at the outer edge of the Bödewadt-like end-wall layer. It appears from their studies, however, that these end-wall-layer instabilities do not influence the spin-down much unless the centrifugal instabilities are somehow eliminated. The main body of this work implies that spin-down is a complex, global problem with at least two instability mechanisms at work that lead to more rapid spin-down, and much more complicated dynamics than could possibly be anticipated from the linear theory or its supposed weakly nonlinear generalization. 

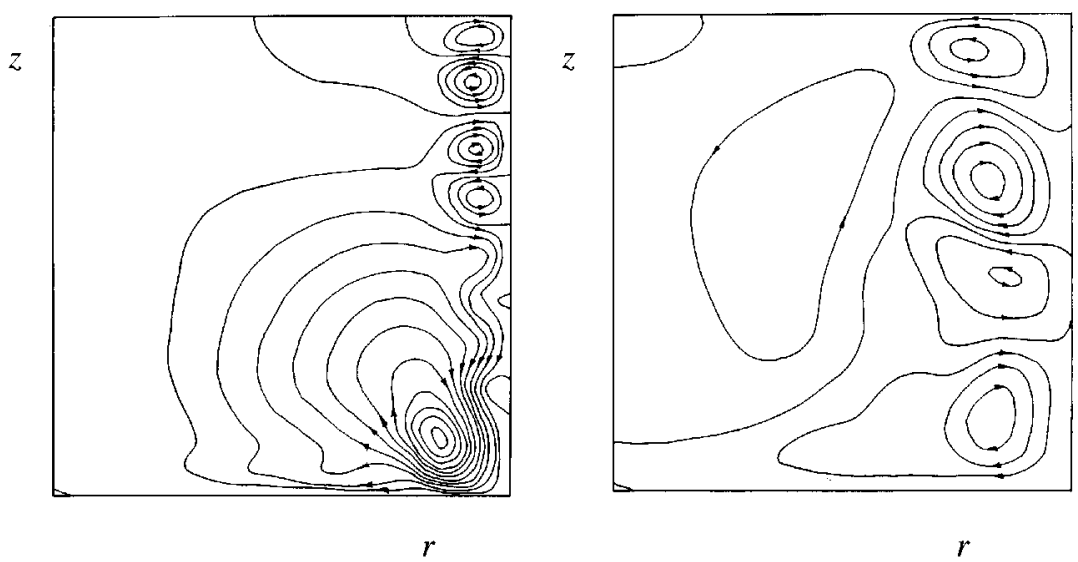

Figure 3 Computed instantaneous streamlines for spin down to rest. $E=2.5 \times 10^{-4}$. (left) $\Omega t=11.24$; (right) $\Omega t=29.9$. Left-hand boundaries correspond to axis of rotation, upper boundaries to symmetry plane. Reproduced from Neitzel \& Davis (1981).

\subsection{Axisymmetric Spin-Over}

In the cases of spin-up from rest and (to a degree) spin-down to rest, the Ekman layers on the lid and bottom are relatively well-behaved (albeit they do play a key role in the overall process). This is borne out by the fact that similarity solutions to the flow above an infinite disk exhibit a well-behaved structure; in the former case they approach, at large times [based on $O\left(\Omega^{-1}\right)$ ], the well-known von Kármán (1921) solution, and in the latter they approach stationary flow. However it is also known that in the general case where the flow far from the disk rotates in the opposite sense to that of the disk itself, although the similarity form of von Kármán is still valid, steady-state solutions to the governing equations do not always exist (dependent on the value of the ratio of the farfield rotation rate to the rotation rate of the disc). In many regions of parameter space the situation is complicated by high degrees of nonuniqueness; a detailed account of this problem is given by Zandbergen \& Dijkstra (1987). The nonexistence of similarity solutions is confined to a negative $[-1.35>\sim \Omega /(\Omega+\Delta \Omega)>\sim-0.16]$ region of parameter space, corresponding to counter-rotations (appropriate therefore to the question of spinover). For instance, what is the effect of changing the rotation rate of the disk such that any (hypothetical) steady state would be in the range of the nonexistence of a steady-state solution. A series of papers (Bodonyi \& Stewartson 1977, Bodonyi 1978, Stewartson et al 1981) showed that in the case of spin-over, three possible scenarios exist. (a) The flow can take on the terminal state of the corresponding steady-state solution, if it exists. (b) The flow can suffer a finite-time breakdown, as elucidated by Bodonyi \& Stewartson (1977) and Stewartson et al (1982). (c) Intriguingly, a limit cycle behavior is possible (Bodonyi 1978). A further possibility 
exists in that certain of these flows may be unstable to nonaxisymmetric modes (in particular those with mode number two), as suggested by the work of Hewitt et al (1999) and Hewitt \& Duck (2000).

The ultimate behavior in contained, spin-over flows remains an unanswered question.

\subsection{Spin-Up in Partially-Filled Containers}

The discussion above, and indeed much of the discussion throughout this review, is directed toward flows in completely filled containers; it is worth making some mention of the spin-up in partially filled containers, i.e. flows involving free surfaces. The seminal paper of Greenspan \& Howard (1963) considers the effects of free surfaces in situations for which the rotation rate is changed by a small amount, compared with the initial rotation rate. There are two significant differences in comparison with the fully enclosed container. First, the viscous effects on the lid are removed so that the interior of the flow extends to the free surface. Second, the change in shape induces a radial motion, thus modifying the spin-up process; Greenspan \& Howard (1963) identified an additional key parameter, the Froude number $F=\Omega^{2} a^{2} / g h$.

An early study on spin-up from rest in partially filled containers was presented by Goller \& Ranov (1968) and later extended by Homicz \& Gerber $(1986,1987)$. These authors presented simple formulae to describe both the transient free-surface shape and the azimuthal velocity profile. Cederlof (1988) considered the spinup process in a container with a parabolic bottom, which was chosen to match the parabolic free surface. This eliminated the effects of vortex stretching. An extensive experimental investigation into free surface effects was performed by Choi et al (1989), whereas a later study (Choi et al 1991) paid particular attention to situations in which the free surface intersects with either or both end-wall discs. More recent theoretical and experimental work on (flat bottomed) containers was published by O'Donnell \& Linden (1991), and a good comparison is found between the two approaches.

\subsection{Spin-Up in Nonaxisymmetric Containers}

There has been a significant amount of work on spin-up in containers that lack axial symmetry, and there are two broad categories of such containers: containers with a nonaxisymmetric depth variation, whose dynamics are radically different because there are no closed geostrophic contours; and containers of uniform depth, but with a noncircular horizontal section. Here we present a necessarily abbreviated view of the status of this work; the interested reader is referred to the more comprehensive overview given by van de Konijnenberg (1995).

In the first category are results reported in Greenspan (1968) on spin-up in a cylindrical container whose lower surface is a plane set at an oblique angle to the rotation axis. For small Rossby numbers, spin-up still occurs on the same timescale as for the constant-depth case, but the circular motion is replaced by a 
set of westward-propagating Rossby waves. Very recent work by Maynes et al (1999) examined the flow inside a circular cylinder that is driven by a rotating bluff body (note, however, that the flow in this study was considered to be turbulent).

In flows of the second category, van Heijst and colleagues have described a combination of careful experiments and analytical solutions for short times. van Heijst (1989) and van de Konijnenberg et al (1996) studied spin-up in circular containers with internal obstructions of a nonaxisymmetric nature; and van Heijst et al (1990), Suh (1994), Henderson et al (1996), and van de Konijnenberg \& van Heijst (1997) examined spin-up in rectangular containers. In both cases, three phases of the motion are distinguishable: $(a)$ on a timescale $\Omega^{-1}$, the establishment of the Ekman layers, as well as an initial uniform vorticity flow pattern induced by the noncircular geometry; $(b)$ the establishment of large vortical patterns in the flow-often quite regular arrays of cells; and $(c)$ the decay of those cells on the $\Omega^{-1} E^{-1 / 2}$ spin-up timescale to leave a rigidly rotating fluid. Figure 4 shows computations for such a spin-up for a particular case; the three regimes are clearly in evidence. The merging of corner vortices into vortices in the central core may or may not take place depending on a number of factors, which include the container shape (van Heijst et al 1990, van de Konijnenberg et al 1989) and the presence or lack of a free upper boundary (van de Konijnenberg \& van Heijst 1997). [Figures very much like that in Figure 4 can also be found in Henderson et al (1996).] The latter effect inhibits the merging of corner vortices into the interior. In the case shown, the initial phase $(a)$ anti-cyclone (see Figure 5, discussed below) decays under the influence of the (phase $b$ ) cyclonic vortices that form out of the boundary-layer vorticity. Each of these cyclonic vortices moves clockwise under the influence of the other, and they merge into a central cyclone-finally decaying on the spin-up scale. (The spin-up time here is $\sim 270 \mathrm{~s}$.) All researchers report streamline patterns that are in excellent agreement with patterns obtained experimentally. Because these flows are strongly two-dimensional even in the nonlinear regime, van Heijst (1989), van Heijst et al (1990), van de Konijnenberg et al (1994), and van de Konijnenberg \& van Heijst (1997) have utilized plots of vorticity versus streamfunction as a gauge of quasi-steady behavior of the core flow on the $\Omega^{-1}$ timescale. The $\omega-\psi$ plots shown in Figure 4 , taken from experiment, are examples.

Interestingly, in all the cases examined by these authors, the initial flow, in the early part of time-regime $(a)$, can be simply analyzed by utilizing the idea that the absolute vorticity is conserved, so that when viewed in the rotating frame of the spun-up fluid, the vorticity is $-2 \Omega$. This implies that one may describe the initial flow state by solving the Poisson equation

$$
\nabla^{2} \psi^{*}=2 \Omega
$$

within the two-dimensional domain of the particular container under consideration. Figure 5 shows excellent agreement between the computed and measured flow field. The complication, of course, which leads to state $(b)$, is that solutions to 

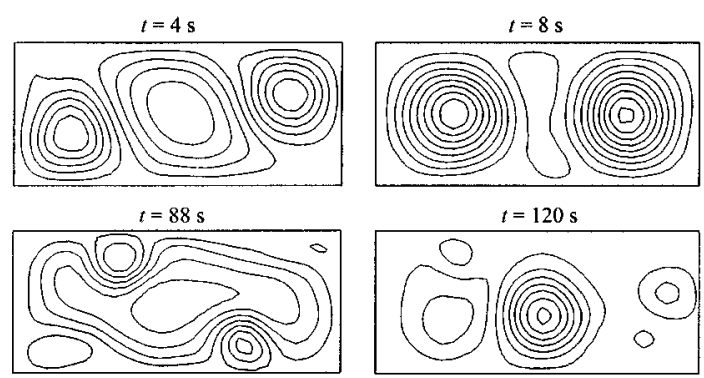

$t=120 \mathrm{~s}$

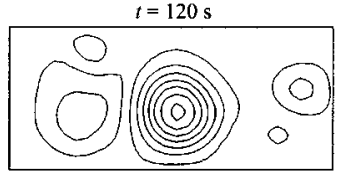

(a)
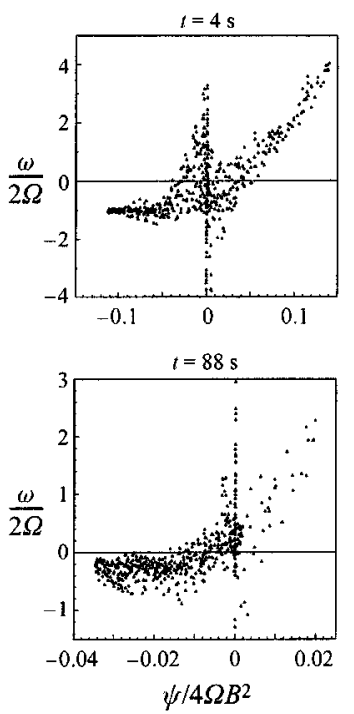
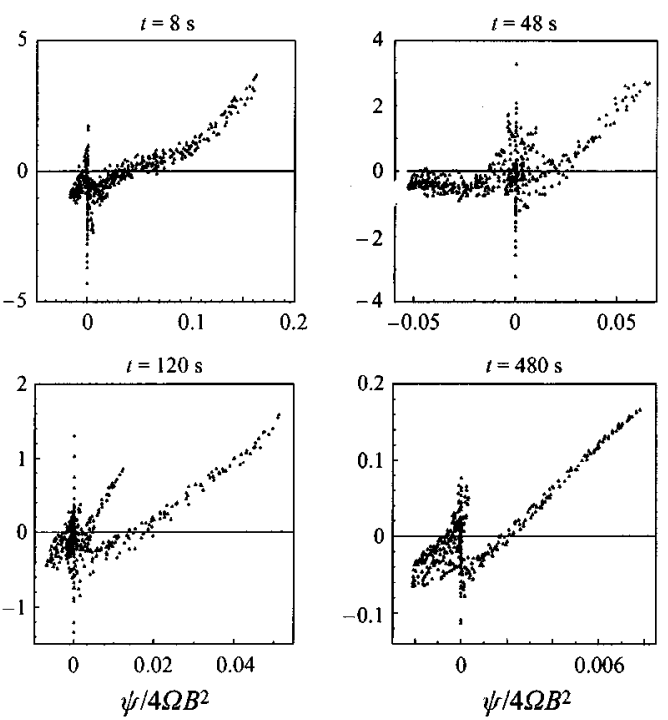

(b)

Figure 4 Spin-up from rest in a rectangular container with aspect ratio 9:4. $E=4.8 \times$ $10^{-6} .2 B$ is the tank width; $(a)$ computations of streamline patterns. Agreement with experiment (not shown here) is excellent; $(b)$ vorticity-streamfunction plots from experiment. Reproduced from van de Konijnenberg \& van Heijst (1997).

Equation 10 slip over the boundaries, generating large vorticity in their vicinity. These boundary layers originate with width $\left(v t^{*}\right)^{\frac{1}{2}}$, and then in certain locations erupt into the fluid interior causing the fluid to break away from the walls and carry vorticity into the interior. Based on the simple ideas for start-up flows, such separation is expected to occur on a timescale of order $\Omega^{-1}$, which is in fact observed in the experiments. Organization into a (sometimes) regular array 


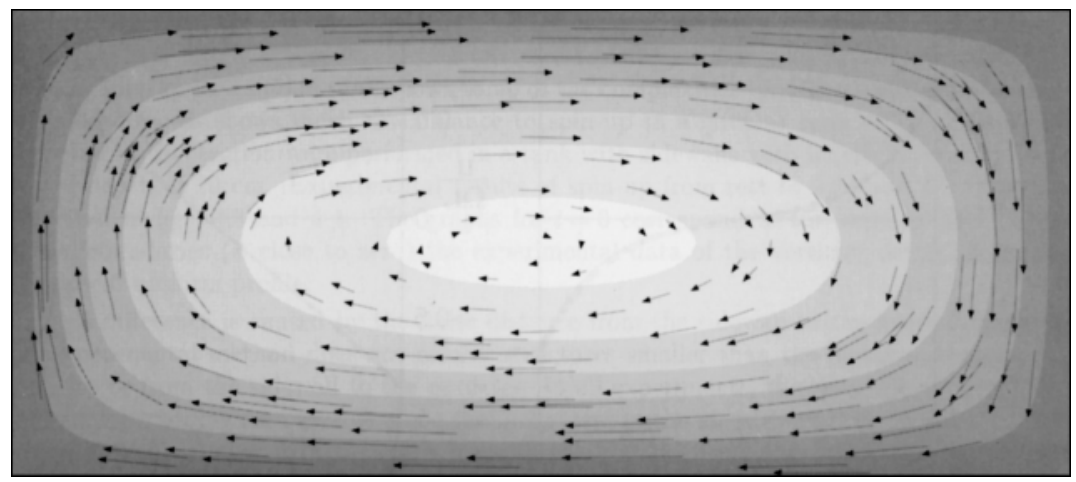

Figure 5 Starting flow spin-up from rest in a rectangular container with aspect ratio 9:4. $E=3.17 \times 10^{-4}$. Comparison of experiment (arrowheads) and theoretical predictions (shaded contours) from Equation 10. Reproduced from van de Konijnenberg \& van Heijst (1997).

of vortices then occurs over a few more rotation periods, and finally decays under the effects of bottom friction. All of the details described are seen in the time sequence of Figure 4.

We conclude that spin-up mechanisms are quite different in axisymmetric and nonaxisymmetric containers: The shedding of boundary-layer vorticity into the core on an $\Omega^{-1}$ timescale obviously cannot occur in an axisymmetric setting. The result is that the initial condition for motion on the spin-up timescale is highly nonuniform in the nonaxisymmetric case.

\section{STRATIFIED FLUIDS}

The combination of stratification and rotation leads to a fascinating and rich assortment of complex physical phenomena and is important for many diverse fields, including numerous industrial applications, as well as atmospheric and oceanographic processes. In this section, we retain the nomenclature of the previous sections on homogeneous flows, and introduce additional parameters and variables, as appropriate. In particular, we follow the usual Boussinesq approximation, including density variation from either thermal or salinity variations, in the buoyancy term only. We ignore any Sweet-Eddington circulations, which can be justified provided $\Omega^{2} r / g \ll 1$. [Studies on this point can be found in Zeipel (1924) and Greenspan (1968).]

New parameters introduced by stratification include a reference density, $\rho_{0}^{*}$, and the diffusivity, $\kappa$. Typically, the density anomaly, $\rho^{*}-\rho_{0}^{*}$, is taken to be small compared to $\rho_{0}^{*}$, so we write

$$
\rho^{*}=\rho_{0}^{*}+\rho_{s}^{*}\left(z^{*}\right)+\rho^{*}\left(\mathbf{r}^{*}, t\right)
$$


with a corresponding decomposition for the pressure, $p^{*}$. The subscript $s$ term denotes the variation due to the basic stratification; in order to make further reasonable progress, we follow most previous studies in taking $\rho^{*} \ll \rho_{s}^{*}$ and $d \rho_{s}^{*} / d z^{*}=$ constant $<0$, the latter condition corresponding to a stable linear stratification.

If we perform the following nondimensionalizations, $\mathbf{r}^{*}=h \mathbf{r}, t=\tau^{*} t, \mathbf{u}^{*}=$ $\epsilon \Omega h \mathbf{u}, p^{*}=2 \Omega \epsilon \rho_{0}^{*} h p$, and $\rho^{*}=2 \Omega \epsilon \rho_{0}^{*} g^{-1} \rho$, and suppose that the initial and final rotation rates of the container are $\Omega$ and $\Omega(1+\epsilon)$, respectively, then under the stated assumptions the equations of motion in a rotating frame are

$$
\begin{gathered}
\nabla \cdot \mathbf{u}=0 \\
\delta_{T} \frac{\partial \mathbf{u}}{\partial t}+2 \mathbf{k} \times \mathbf{u}+\epsilon \mathbf{u} \cdot \nabla \mathbf{u}=-\nabla \rho-\rho \mathbf{k}+E \nabla^{2} \mathbf{u},
\end{gathered}
$$

and

$$
\delta_{T} \frac{\partial \rho}{\partial t}+\epsilon \mathbf{u} \cdot \nabla \rho-S \mathbf{u} \cdot \mathbf{k}=\frac{E}{\sigma} \nabla^{2} \rho .
$$

Three additional nondimensional parameters pertinent to rotating stratified flows have appeared: the Schmidt number, $\sigma=\nu / \kappa$; the Burger number $S=N^{2} / \Omega^{2}$, where $N=\left[-\left(g / \rho_{0}^{*}\right)\left(\partial \rho_{s}^{*} / \partial z^{*}\right)\right]^{1 / 2}$ is the Brunt-Väisälä frequency (taken to be constant in line with our comments above); and a reciprocal timescale parameter $\delta_{T}$ $=1 /\left(\Omega \tau^{*}\right)$. They may be chosen as appropriate. Based on the foregoing analysis, we expect that $\delta_{T}$ is $E^{\frac{1}{2}}$-at the very least for $S$ sufficiently small.

Much of the work in rotating and stratified spin-up has concentrated on the small Rossby number limit (corresponding to relatively small changes in the rotation of the container), i.e. $\epsilon \ll 1$, which leads to a linearized problem, as in the homogeneous case. This limit is considered first (in the following section), before we extend our discussion to order-one Rossby numbers and the subsequent nonlinear system that results.

\subsection{Linear, Axisymmetric Spin-Up}

The history of stratified spin-up in rotating containers can be traced to Ekman (1906), who performed a series of experiments using a light aluminum vane in the core of the flow to measure the rate of transfer of momentum between the Ekman layer on the base of the container and the interior of the fluid. His primary conclusion was correct: Stratification serves to inhibit the penetration of boundary vorticity into the main body of the fluid. Pettersson (1931) repeated the experiments of Ekman and confirmed Ekman's conclusion regarding the transfer of momentum between the boundaries and the core of the flow. But he also observed a secondary circulation, caused by the spin-up, and suggested that the transfer of vorticity out of the boundary layer created this circulation, as opposed to a vertical diffusion process. However, the picture of the secondary flow described by Pettersson (1931) was flawed: He claimed that the flow is thrown outward near the bottom of the container, flows up the side wall, turns horizontally inward 
into the core of the flow, and then flows into the lower boundary layer along a thin layer near the center of the container. We show below that this is the wrong picture.

Early attempts at a theoretical description of (linear) stratified spin-up led to a series of papers, and a debate over the correct nature of the flow. Holton (1965) considered the spin-up of a (thermally) stratified Boussinesq fluid in a right circular cylinder. He claimed (correctly) that the Ekman layers take on basically the same form as in the nonstratified case. (Thermal boundary layers may also occur, but these play a passive role in the overall dynamics.)

Pedlosky (1967) reexamined the problem, in particular investigating the effects of the sidewall boundary, which are crucial for spin-up. He showed correctly that when the side wall is thermally insulated, the $E^{\frac{1}{4}}$ layer is unable to transport the $\left[O\left(E^{\frac{1}{2}}\right)\right]$ mass flux discharged from the Ekman layer at the corner. This led Pedlosky to the (erroneous) conclusion that because there can be no inflow from the $E^{\frac{1}{4}}$ layer into the core (as there is in the homogeneous case), spin-up does not occur at all for configurations with a no-penetration sidewall condition on the density.

Holton \& Stone (1968) pointed out certain inconsistencies with some of Pedlosky's (1967) scaling arguments, and also noted that his results were at variance with experimental observations also presented by Holton (1965). The controversy was eventually resolved by Walin (1969) and Sakurai (1969), who showed that neither Holton nor Pedlosky was totally correct: Holton predicted the qualitative flow pattern correctly but missed the inability of the (insulated) sidewall layer to transport fluid; Pedlosky, while correctly analyzing the sidewall layer, falsely concluded that horizontal boundary layers do not exist, and "the interior spins up by a strictly diffusive process." In reality, there is a (source-like) eruption of fluid in the corners of the tank.

We have detailed here some of the early history and fundamental features of stratified spin-up and illustrated some of the subtleties that occur, even in the linearized (i.e. small Rossby number) regime. Below, we present a brief resumé of the solution structure, following Walin (1969) and Sakurai (1969). We show that, in fact, two different choices of inverse timescale, $\delta_{T}=E^{\frac{1}{2}}$ and $E$, are important to the spin-up description.

For $\delta_{T}=O(1)$, i.e. a timescale comparable with the rotation time of the container, a simple Ekman layer spin-up process occurs on the horizontal walls prior to any substantial response of the fluid in the main body of the container, as in the homogeneous case.

We now consider the central issue, that of the boundary layer on the side wall. Using Equation 14 and writing the boundary-layer scale in the form $\xi=$ $E^{-\frac{1}{2}}(r-a)$ (the $E^{\frac{1}{2}}$ thickness of this layer is demanded by consistency with the $z$ momentum equation), then the leading-order terms are

$$
-S w=\frac{1}{\sigma} \rho_{\xi \xi}
$$


Integrating Equation 15 across the sidewall boundary layer, so long as the timescale is longer than $O(1)$, leads to

$$
\left.-S \int_{-\infty}^{0} w d \xi=\frac{1}{\sigma} \rho_{\xi}\right]_{-\infty}^{0} .
$$

The lower limit of the right-hand term must be zero if proper matching with a core region (in which $w$ is relatively small compared with that in the sidewall layer) is to be achieved, whereas the upper limit must also be zero in the insulated-wall case. Thus, since the right-hand side of Equation 16 is zero, we conclude that the net vertical fluid transport is zero. The inevitable conclusion (Walin 1969) is that the radially transported fluid in the Ekman layers must erupt out of the corners where the Ekman layers meet the outer wall. This eruption must occur on a radial scale of size $E^{\frac{1}{2}}$, and so, on an outer scale, the eruption is singular and most easily modeled by a delta function (see Spence et al 1992). A schematic of the resulting meridional-plane streamlines is shown in Figure 6.

Taking the limits $E, \delta_{T} \rightarrow 0$ [but $E=o\left(\delta_{T}\right)$ ] in Equations 13 and 14, it turns out that the leading-order gauge functions for $v, p$, and $\rho$ are 1, but the meridional circulation is slow as in the homogeneous case, so that $(u, w)=\delta_{T}\left(u_{0}, w_{0}\right)$. The first terms in the outer expansion satisfy the equations

$$
\begin{gathered}
-2 v_{0}+\frac{\partial p_{0}}{\partial r}=0, \\
\frac{\partial v_{0}}{\partial t}+2 u_{0}=0, \\
\frac{\partial p_{0}}{\partial z}+\rho_{0}=0, \\
\frac{\partial \rho_{0}}{\partial t}-S w_{0}=0,
\end{gathered}
$$

and

$$
\frac{1}{r} \frac{\partial\left(r u_{0}\right)}{\partial r}+\frac{\partial w_{0}}{\partial z}=0 .
$$

It is convenient to deal with the pressure time derivative as a fundamental quantity, so we write $\phi=-\partial p_{0} / \partial t$. In this case, the equations reduce to the following:

$$
\begin{aligned}
& u_{0}=\frac{1}{4} \frac{\partial \phi}{\partial r}, \quad w_{0}=\frac{1}{S} \frac{\partial \phi}{\partial z}, \\
& \frac{1}{r} \frac{\partial}{\partial r}\left[r \frac{\partial \phi}{\partial r}\right]+\frac{4}{S} \frac{\partial^{2} \phi}{\partial z^{2}}=0 .
\end{aligned}
$$




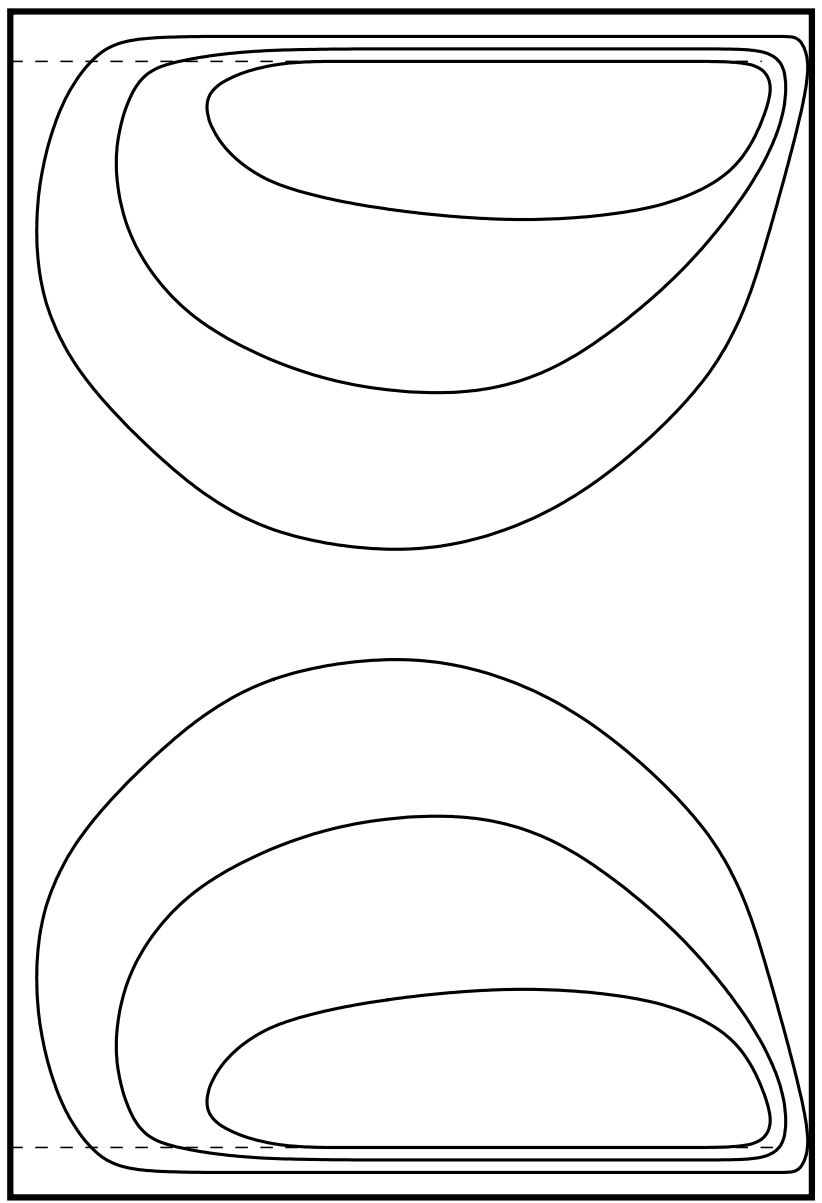

Figure 6 Schematic of meridional-plane streamlines for stratified spin-up. The left-hand boundary is the container centerline.

At the outer wall, because we have shown there is no vertical fluid transport in the sidewall layer, there is also no fluid entrainment by the layer, and hence $\partial \phi / \partial r=0$ at $r=a$. At the upper and lower boundaries, we impose Ekman compatibility conditions as in the homogeneous case, which also indicates that $\delta_{T}=E^{\frac{1}{2}}$. Thus,

$$
\begin{aligned}
& w_{0}=\frac{1}{2 r}\left[\frac{\partial\left(r v_{0}-r^{2}\right)}{\partial r}\right]+\frac{a}{2} \delta(r-a) \quad \text { on } \quad z=0, \\
& w_{0}=-\frac{1}{2 r}\left[\frac{\partial\left(r v_{0}-r^{2}\right)}{\partial r}\right]+\frac{a}{2} \delta(r-a) \quad \text { on } \quad z=1 .
\end{aligned}
$$


The delta functions are representations of the source-like eruptions (or point sinks) of the Ekman transport at the outer corners of the container. These conditions are of course applied impulsively at $t=0+$, so there is a temporal step function multiplying the spatial delta function terms.

The problem is most easily solved by means of a Fourier-Bessel series,

$$
\phi=\sum_{n=1}^{\infty} A_{n}(z, t) J_{0}\left(\alpha_{n} r / a\right),
$$

where the $\left\{\alpha_{n}\right\}$ are the zeroes of $J_{0}^{\prime}(x)$, in order to satisfy the condition on $r=1$. Each Fourier mode decays exponentially, with time constant given by $\tanh \left(\beta_{n} / 2\right) / \beta_{n}$, where $\beta_{n} \equiv \alpha_{n} S^{1 / 2} /(2 a)$. Letting $t \rightarrow \infty$ in this solution gives the final result for this phase of the spin-up,

$$
v_{0} \rightarrow-a \sum_{n=1}^{\infty} \frac{2 \cosh \left[\beta_{n}\left(z-\frac{1}{2}\right)\right] J_{1}\left(\alpha_{n} r / a\right)}{\alpha_{n} J_{0}\left(\alpha_{n}\right) \cosh \left(\beta_{n} / 2\right)} \quad \text { as } \quad t \rightarrow \infty .
$$

This series is easily verified as precisely the $J_{1}$ Fourier-Bessel series for $r$ if $z$ is set to either 0 or 1 , and so

$$
v_{0} \rightarrow r \quad \text { as } \quad t \rightarrow \infty \quad \text { on } \quad z=0,1 .
$$

It is important to remember that $\infty$ for this timescale means only many spinup times, and the above result serves as an initial condition for the subsequent (longer) temporal development. Notice that on the spin-up timescale, the fluid is fully spun up near the horizontal boundaries, but not in the interior! The final stage of interior spin-up occurs on a longer, diffusive timescale corresponding to the timescale parameter $\delta_{T}=E$. Returning to Equations 12-14, with this choice of $\delta_{T}$, we obtain the set of equations for the interior motion on this longer timescale as

$$
\begin{gathered}
-2 \hat{v}+\hat{p}_{r}=0, \\
\hat{v}_{\tau}+2 \hat{u}=\nabla^{2} \hat{v}-\frac{\hat{v}}{r^{2}}, \\
\hat{p}_{z}=-\hat{\rho}, \\
\hat{\rho}_{\tau}-S \hat{w}=\frac{1}{\sigma} \nabla^{2} \hat{\rho},
\end{gathered}
$$

and

$$
\frac{1}{r} \frac{\partial(r \hat{u})}{\partial r}+\frac{\partial \hat{w}}{\partial z}=0
$$


where hatted variables denote the leading-order quantities on this timescale, and $\tau$ now denotes this scaled time. Again, a Fourier-Bessel series representation for $\hat{v}$ takes the form

$$
\hat{v}=r+\sum_{n=1}^{\infty} C_{n}(z, \tau) J_{1}\left(\alpha_{n} r / a\right)
$$

whereas other hatted quantities may be expressed in a similar series. The solution can be obtained again in the form of a Fourier-Bessel series, which takes a particularly simple form in the case of the large-Schmidt-number limit $(\sigma \rightarrow \infty)$,

$$
C_{n}=\frac{8 a}{\alpha_{n} J_{0}\left(\alpha_{n}\right)} \sum_{\ell=0}^{\infty} \frac{(-1)^{\ell} \beta_{n}^{2} \cos \left[(2 \ell+1) \pi\left(z-\frac{1}{2}\right)\right]}{(2 \ell+1) \pi\left[(2 \ell+1)^{2} \pi^{2}+\beta_{n}^{2}\right]} e^{-s_{n \ell} \tau},
$$

where

$$
S_{n \ell}=\frac{(2 \ell+1)^{2} \pi^{2}+\alpha_{n}^{2} / a^{2}}{(2 \ell+1)^{2} \pi^{2}+\beta_{n}^{2}} \beta_{n}^{2} .
$$

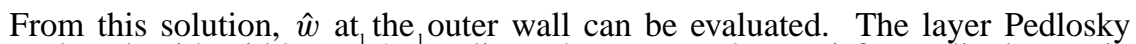
analyzed, with width $\sim E^{\frac{1}{2}} / \sigma^{\frac{1}{4}}$, adjusts the nonzero $\hat{w}$ to satisfy no-slip, but again the profile is oscillatory, with no net transport. At these large $\sigma$ values, Equations 33 and 34 fail in regions near the upper and lower boundaries of height $\sigma^{-\frac{1}{4}}$; in these zones, the final stage of spin-up occurs on a still longer timescale of $O\left(E^{-\frac{1}{2}} \sigma^{\frac{1}{2}}\right)$.

St-Maurice \& Veronis (1975) used multiscaling analysis to consider three disparate timescales (considering both the homogeneous and stratified cases). Related work on various limiting cases has also been carried out; Sakurai et al (1971) and Clark (1973) presented results for small Prandtl numbers, whereas Allen (1971) and Siegmann (1971) investigated the limit of weak stratification.

\subsection{Nonlinear, Axisymmetric Spin-Up}

Early laboratory experiments on impulsively started spin-up flows inside closed circular cylinders were performed by Holton (1965), McDonald \& Dicke (1967), and Modisette \& Novotny (1969). Results from these studies agreed qualitatively with linear theory (detailed above) but were of insufficient accuracy for a detailed quantitative comparison. More accurate experimental studies were conducted by Buzyna \& Veronis (1971), Saunders \& Beardsley (1975), and Lee (1975). Buzyna $\&$ Veronis (1971) used a salt-stratified solution and photographed the motion of fluid dye to measure the angular displacement of the fluid, whereas Saunders \& Beardsley (1975) used a thermally stratified fluid to measure the temperature field with an array of thermistors. Lee (1975) also used a thermally stratified fluid, but instead used a laser Doppler velocimeter (LDV) measuring technique. These latter three studies clearly showed the decay rate was faster than that predicted by the linearized theory of Walin (1969). Later experiments by Greenspan (1980) confirmed the intricate nature of stratified spin-up. In addition to observing a cylindrical shear wave-similar in nature to that described by Wedemeyer (1964) for homogeneous 
fluids, but bowed-he also observed instabilities in the sidewall layer that result in fluid mixing, a process that is particularly vigorous in corner regions.

The first numerical experiments on impulsively started (thermally) stratified spin-up in a circular container were performed by Barcilon et al (1975), who solved the Navier-Stokes equations (using finite-difference techniques). These calculations confirmed prior experimental conclusions that the flow perturbations decay faster than predicted by Walin. Intriguingly Barcilon et al (1975) showed that nonlinearity was not the cause of this discrepancy, but postulated that the discrepancy was due to the corner jet, which oscillates between the near-horizontal and near-vertical directions. A number of studies, including St-Maurice \& Veronis (1975), have suggested this discrepancy is due to diffusive effects; certainly the importance of these effects has been illustrated on the long $t=O\left(E^{-1}\right)$ timescale. Later, numerical calculations were carried out by Hyun et al (1982), extending the model (and basic numerical scheme) of Warn-Varnas et al (1978) to incorporate the effects of (thermal) stratification. Once again, these later computations agreed well with experimental data [careful comparison with that of Lee (1975) was undertaken], but again substantial discrepancies were found when compared with the results of Walin (1969), in particular with regard to the $E^{-\frac{1}{2}} \Omega^{-1}$ timescale. Note from Figure 7 that the experiments and the full computations both show much more rapid decay than the results from linearized theory. The effects of nonlinearity were again investigated but ruled out as the cause of the disagreement. Hyun et al (1982) examined thoroughly the possible sources of the discrepancy between theory and experiment; they concluded that the absence in the Walin theory regarding the modeling of the diffusive timescale behavior is responsible. Figure 8 , which includes diffusive timescale results from the previous section, certainly lends credence to that position. Other numerical investigations into various aspects of the (thermally stratified) problem were conducted by Hyun (1982, 1983, 1984), and more recently Hyun \& Park (1992) studied the spin-up from rest of a compressible fluid in a rotating cylinder, where they concluded that viscous effects are of greater significance than those in the incompressible regime.

Beardsley et al (1979) undertook a series of laboratory, numerical, and theoretical studies on the (thermally) stratified flow inside a circular cylinder, in which the rotation rate of the container was increased linearly in time, from some initial rotation rate. Intriguingly, in contrast to the instantaneous spin-up results, use of essentially the same model as that of Walin (1969) and Sakurai (1969) gives good agreement between the reported asymptotic and experimental results.

\section{STRATIFIED SPIN-UP AND SPIN-DOWN IN CONICAL CONTAINERS}

A number of authors have studied the flow of rotating, stratified viscous flows over topography, i.e. when a flow boundary is neither parallel nor perpendicular to gravity. In these circumstances the fluid may have isopycnals that intersect with the 

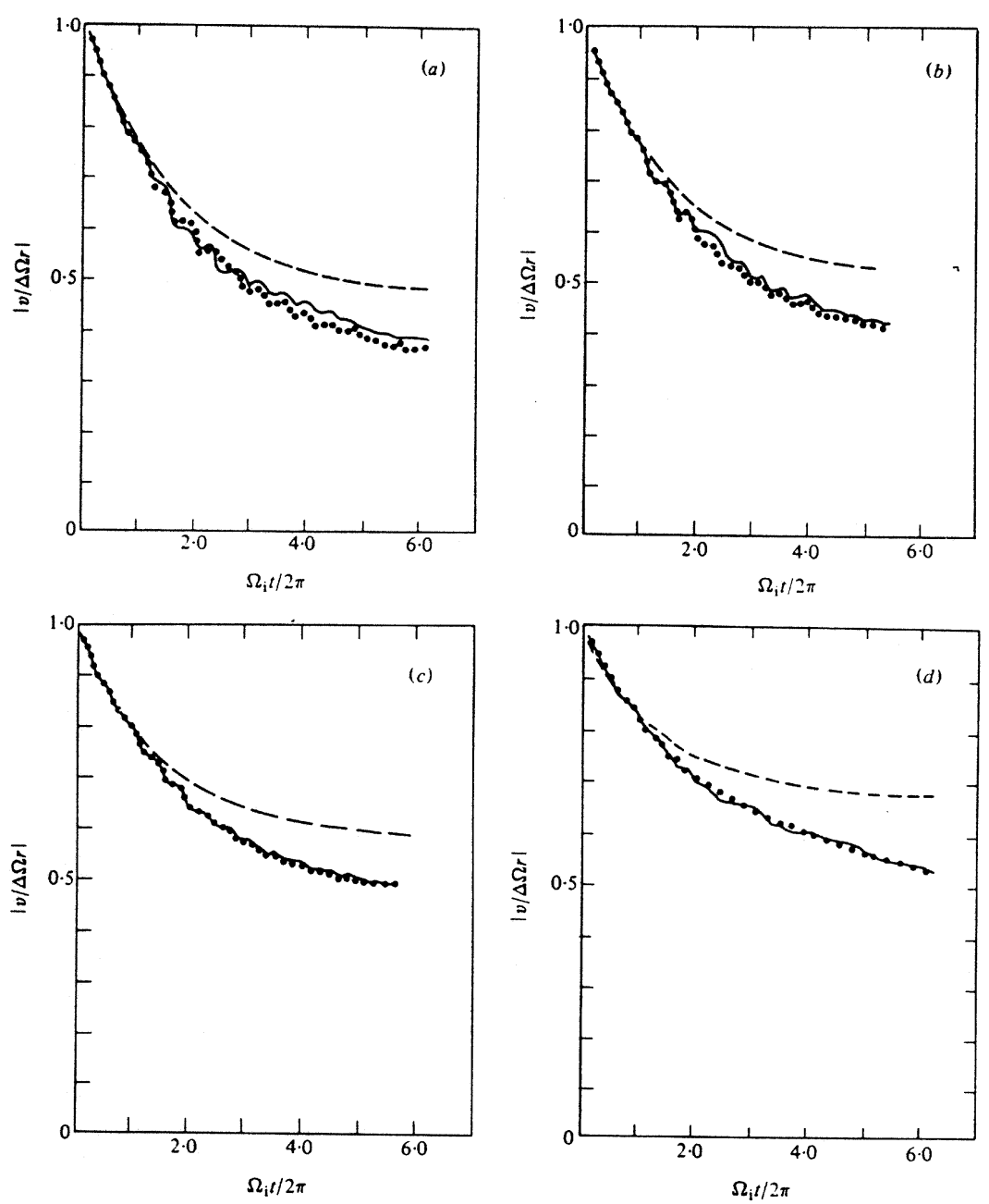

Figure 7 Comparison of spin-down results for $S=0.960, E=2.89 \times 10^{-3}, \epsilon=0.222$. Dots are laser measurements, solid lines are numerical results, and dashed lines are (linear) theoretical results. $z^{*} / h=0.5$ and (a) $r^{*} / a=0.30$, (b) 0.39 , (c) 0.52 , (d) 0.64 . Reproduced from Hyun et al (1982).

boundary, leading to density gradients on the boundary that can then dramatically alter the characteristics of the boundary layer on such surfaces. Thorpe (1987) obtained a linearized (small Rossby number) solution for a steady sloping wall boundary layer of this type and found that such a solution only leads to an overall steady boundary-layer solution under very exceptional circumstances. This is in direct contrast to Ekman layers that form on horizontal or vertical boundaries, for which a steady-state solution is assured. Later, MacCready \& Rhines (1991) 


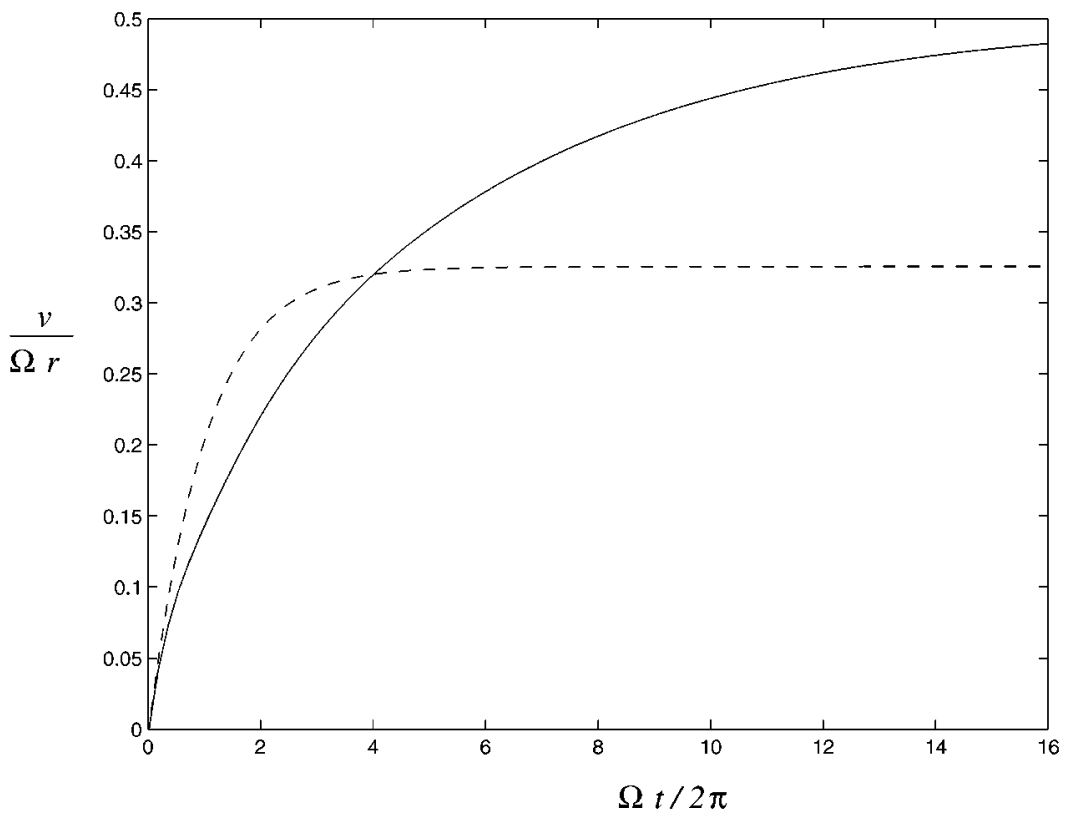

Figure 8 Swirl velocity during large- $\sigma$ stratified spin-up at $r=0.5, z=0.5$. - -, The Walin solution; - , composite of the Walin solution and Equations 33 and 34. For the case shown, $S=2.0, a=1$, and $E=2.89 \times 10^{-3}$.

incorporated unsteady effects into Thorpe's (1987) model, and determined that boundary layers of this type generally do not develop toward a steady state (on timescales long compared to the rotation time, $1 / \Omega$ ). This provides clear evidence of a distinction between flows with sloping walls and those with either horizontal or vertical boundaries.

Duck et al (1997) and Hewitt et al (1999) have considered various aspects of nonlinear spin-up in conical containers, effectively extending the work of MacCready \& Rhines (1991) to finite Rossby numbers. Below we present the details of this nonlinear work and indicate the links to, and salient features of, the work of MacCready \& Rhines (1991).

In this nonlinear case, it is advantageous to use an inertial (nonrotating) frame of reference, in particular using spherical polar coordinates. A sketch of the coordinate system is shown in Figure 9. We suppose $\Omega a\left(v_{r}^{\prime}, v_{\theta}^{\prime}, v_{\phi}^{\prime}\right)$ to be the dimensional velocity components in the $\left(r^{\prime}, \theta^{*}, \phi\right)$ directions, where $\theta^{*}$ is the polar angle, $\phi$ the azimuthal angle, and $a r^{\prime}$ the distance from the cone apex, with $a$ being some measure of the scale of the container. The perturbation density, connected to $\rho^{*}$ in Equation 11 above, is $\frac{\rho o}{g} B^{\prime}$. The boundary-layer coordinate $\theta$ is defined by

$$
\theta^{*}-(\pi / 2-\alpha) \equiv E^{\frac{1}{2}} \theta,
$$

where $\frac{\pi}{2}-\alpha$ is the angle the slope makes with the horizontal. Again, an Ekman 


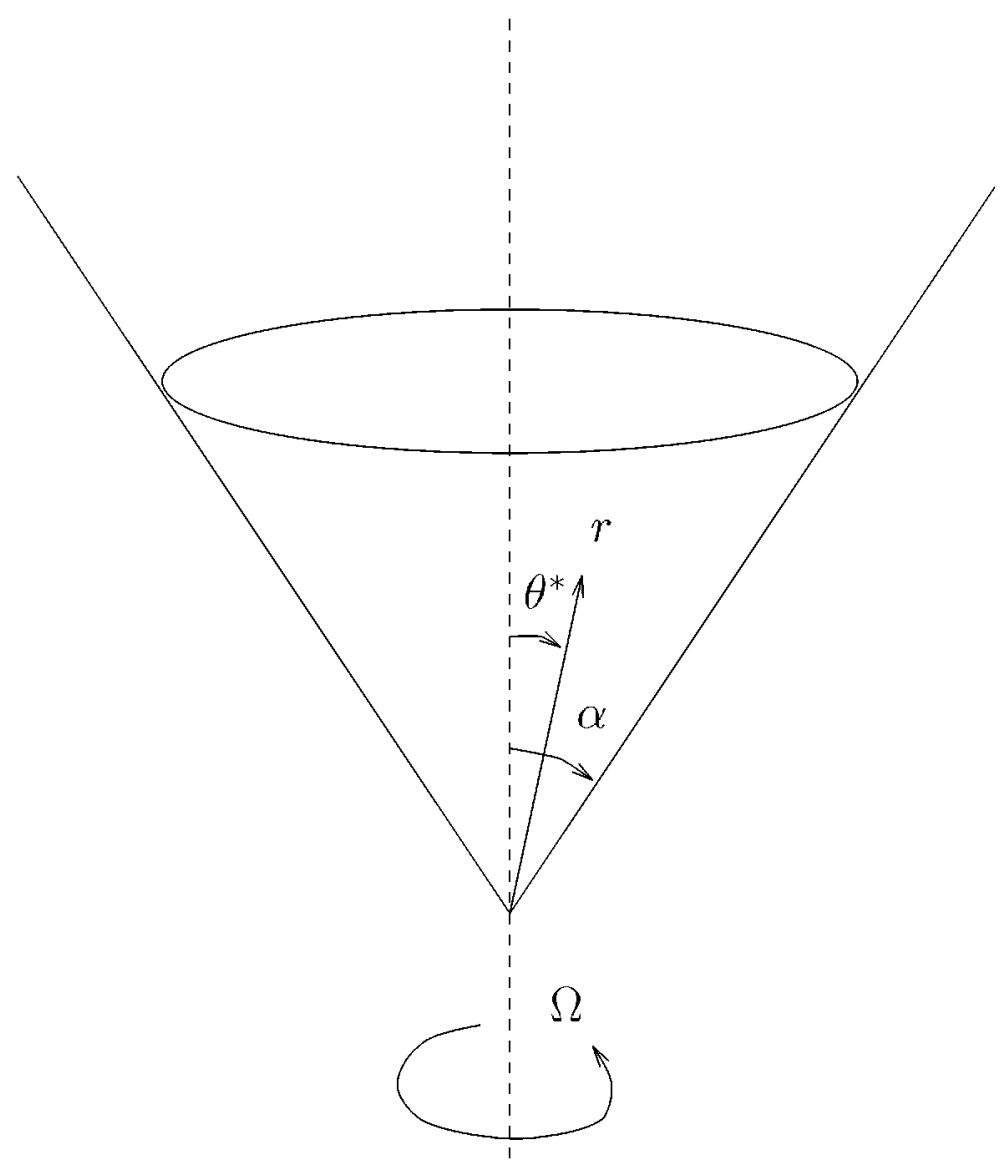

Figure 9 Schematic of conical container configuration.

number, $E \equiv v / \Omega a^{2}$, is taken to be small. Following standard boundary-layer practice, we introduce a scaled normal velocity component $\hat{v}_{\theta}^{\prime}=E^{-\frac{1}{2}} v_{\theta}^{\prime}$. We make the usual Boussinesq and boundary-layer approximations, and seek solutions like those for the classical homogeneous flow above a rotating disk,

$$
\begin{gathered}
v_{\phi}^{\prime}=r^{\prime} \hat{W}(\Theta, t)+\ldots, \\
v_{r}^{\prime}=r^{\prime} \hat{U}(\Theta, t)+\ldots, \\
v_{\theta}^{\prime}=\hat{V}(\Theta, t)-\Theta \hat{U}(\Theta, t)+\ldots, \\
p^{\prime}=r^{\prime 2} \hat{P}(\Theta, t)+\ldots,
\end{gathered}
$$


and

$$
B^{\prime}=r^{\prime} \hat{B}(\Theta, t)+\ldots
$$

where $\Theta=r^{\prime} \theta=O(1)$.

This form of solution is entirely admissible provided $r^{\prime} \gg E^{\frac{1}{2}}$, i.e. locations not in the immediate vicinity of the cone apex (also ignoring any finite container effects, e.g. free surfaces). Because of the sloping boundary, the nonpermeability boundary condition on the density leads to the boundary condition on the density perturbation, and $\hat{B}$ is

$$
\left.\hat{B}_{\Theta}\right|_{\Theta=0}=-E^{\frac{1}{2}} S \cos \alpha .
$$

Because $\hat{B}$ is $O(1)$ in the solutions investigated, this order $E^{1 / 2}$ term can be neglected for small Ekman number.

The number of parameters in the problem may conveniently be reduced by two by introducing the following transformation:

$$
\begin{aligned}
& B^{*}=2 \hat{P}+\hat{B} \sin \alpha, \\
& S^{*}=S \sin ^{2} \alpha+2 \hat{P},
\end{aligned}
$$

and this leads to the central system

$$
\begin{gathered}
2 \hat{U}+\hat{V}_{\Theta}=0 \\
\hat{W}_{t}+2 \hat{U} \hat{W}+\hat{V} \hat{W}_{\Theta}=\hat{W}_{\Theta \Theta} \\
\hat{U}_{t}+\hat{U}^{2}+\hat{V} \hat{U}_{\Theta}-\hat{W}^{2}=\hat{U}_{\Theta \Theta}-B^{*}
\end{gathered}
$$

and

$$
B_{t}^{*}+\hat{V} B_{\Theta}^{*}-S^{*} \hat{U}+\hat{U} B^{*}=\frac{1}{\sigma} B_{\Theta \Theta}^{*}
$$

The boundary conditions are

$$
\begin{aligned}
& \hat{W}=1, \hat{U}=0, \hat{V}=0, B_{\Theta}^{*}=0 \quad \text { on } \quad \Theta=0, \quad t>0, \\
& \hat{U} \rightarrow 0, \hat{W} \rightarrow \hat{W}_{e}, B^{*} \rightarrow B_{e}^{*}=\hat{W}_{e}^{2} \quad \text { as } \quad \Theta \rightarrow-\infty
\end{aligned}
$$

With the transformations above, only three independent parameters characterize the solution, namely a modified Burger number, $S^{*}$; the ratio of the swirl velocity at the layer edge to the wall swirl velocity, $\hat{W}_{e}$; and the Prandtl/Schmidt number, $\sigma$. Note that nonstratified flows correspond to the case $S^{*}=\hat{W}_{e}^{2}$. (Statically stable zones correspond to $S^{*}>\hat{W}_{e}^{2}$, and statically unstable zones to $S^{*}<\hat{W}_{e}^{2}$.) 
MacCready \& Rhines (1991) performed a numerical study of a linearized version of this system, together with an approximate asymptotic treatment of the governing equations; their work corresponds to $\hat{W}_{e}$ very close to 1 . Duck et al (1997) used a detailed Laplace transform treatment on the linearized problem, and confirmed the general lack of a steady state; this showed that the special solution found by Thorpe (1987) is attained as an ultimate state only in a very narrow region of parameter space for which the difference between the edge and wall azimuthal velocities is $o\left(E^{2}\right)$.

Duck et al (1997), focusing on moderate values of Prandtl number $\sigma$, found a variety of flow behaviors by solving the full nonlinear, initial value system. In some situations they found that as time progresses, the flow inside the boundary layer does develop into a steady state, but one that is quite distinct from that obtained by Thorpe (1987). In other situations, the flow develops a finite-time singularity, with the wall shear stresses becoming unbounded at a finite value of time. In other regimes, although the boundary layer develops a steady inner region, an outer region also forms, whose thickness grows without bound as time increases. In a later study by Hewitt et al (1999), for the larger values of Schmidt number, $\sigma$, pertinent to saline solutions, still another behavior was identified: a second class of finite-time breakdown, linked to the nonlinear development of instabilities (of continuous spectrum type). Figure 10 conveniently summarizes these ideas, giving a global picture the flow behavior over a wide range of spin-up/down parameter space for the case $\sigma=700$. Note that the finite-time breakdown zone (ii) corresponds to the latter class of breakdown, zone (i) to the former class. The complicated nature of the steady-state flow was further confirmed by Hewitt et al (1999), who found much solution nonuniqueness, together with zones of nonexistence.

Hewitt et al (1999) made a comparison between experiment and nonlinear theory. The experiments used a saline fluid in a cone that was seeded with (small) particles, visualized by illuminating the particles with a horizontal light sheet, and then monitored with a CCD camera. Density measurements were also made. A comparison between experiment and (nonlinear) theory is shown for a spin-up example in Figure 11 and indicates a good comparison.

In the case of spin-down, the comparison between theory and experiment is poor. Figure 12 shows such a comparison, and indicates large discrepancies. Figure 13 shows typical velocity vectors taken from experiment, and points to part of the explanation for these discrepancies, the formation of a strong nonaxisymmetric structure within the flow. The reason for this is likely linked to nonaxisymmetric centrifugal instabilities of the basic state. Indeed Hewitt et al (1999) have found a steady nonaxisymmetric state to the nonstratified equations of motion (in fact an exact solution of the Navier-Stokes equations), clearly pointing the way to such a possibility.

Other shaped conical containers (with radii taking on a general algebraic, rather than linear, form) were studied by Hewitt et al (1998). This study indicates that the 


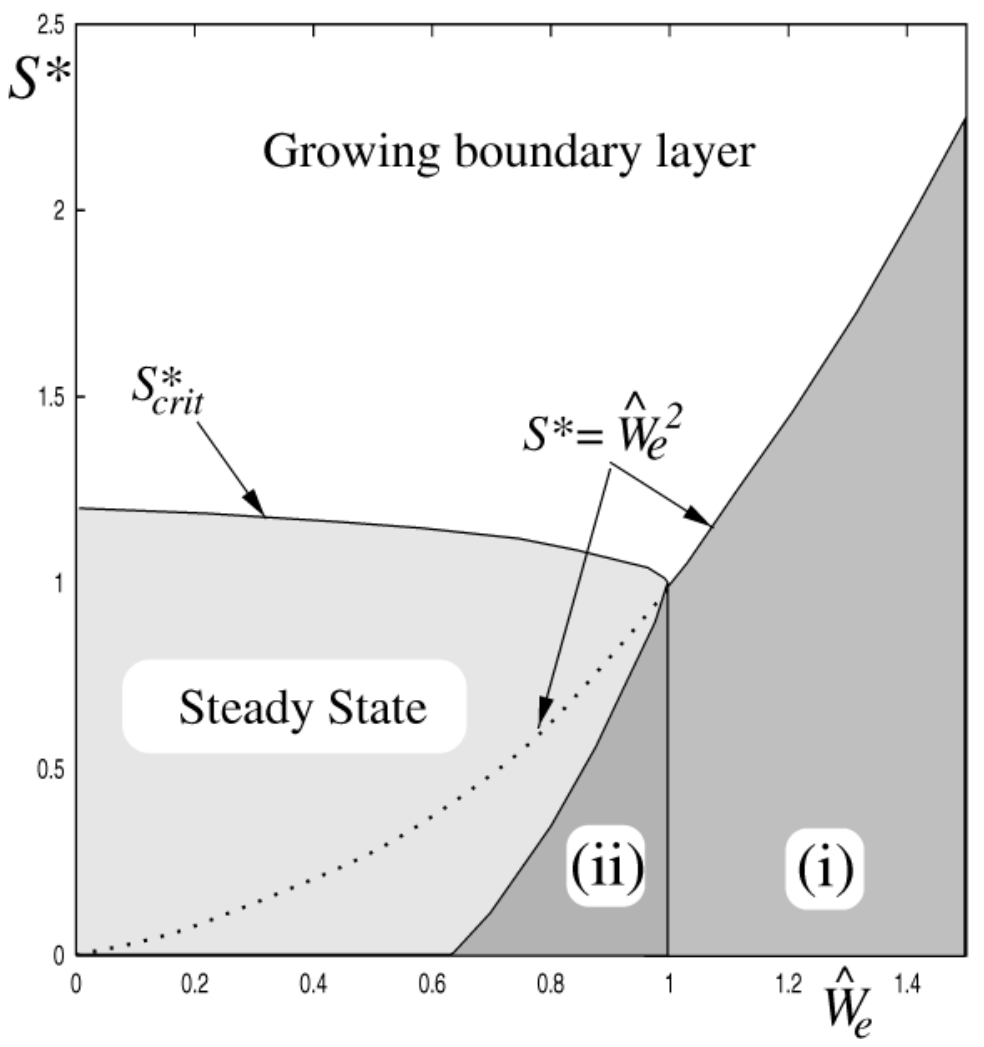

Figure 10 Nature of flow behaviors, $\sigma=700$.

straight cone is likely to be the most interesting, with the most physical interactions between processes.

To summarize the work on rotating, stratified flows involving sloping boundaries, perhaps the most significant observation is the ability of the associated Ekman layers to breakdown, even in the linearized (small Rossby number) case. In rotating, stratified flows involving nonsloping geometries, at least on the $t^{*}=O\left(\frac{1}{\Omega}\right)$ timescale, the Ekman layer is relatively passive (although the layer does promote a flow outward radially that leads to the difficulties mentioned above). In the case of sloping boundaries there is the strong possibility of either breakdown within this timescale, leading presumably to a burst of vorticity into the main body of the fluid (although such behaviors seem to be confined to zones of statically unstable stratification), or a boundary layer that grows without bound, contaminating the core of the flow with viscosity in a relatively short time [i.e. $O(1) \ll \Omega t^{*} \ll$ $\left.O\left(E^{-\frac{1}{2}}\right)\right]$. 


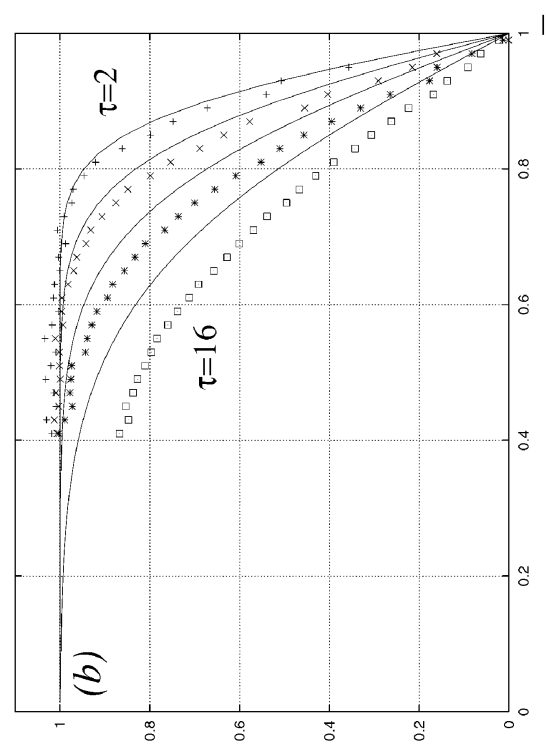

11 I

$\sim \dot{\mathrm{c}}$.

क舫

苍 $\mathrm{G}$

흐를

艄

a

롱 로

总

$\frac{\infty}{\infty} \geqslant$

壱

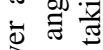

突

的 包

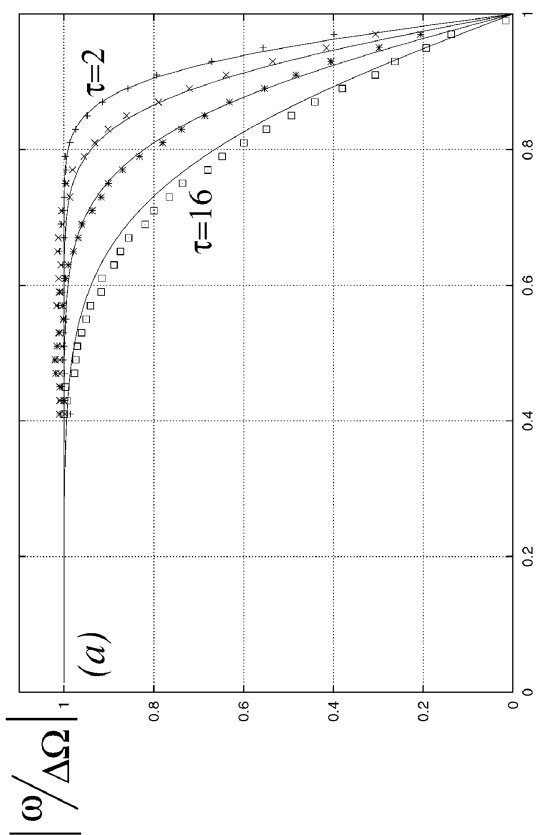

药

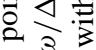

茞 $\frac{3}{4}$

己

ฮี

은

离造造方

ఖ

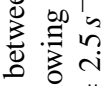

放

节令

है

ठै

\& $\|$ is

$=\stackrel{t}{0} \frac{n}{k}$

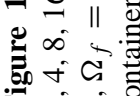




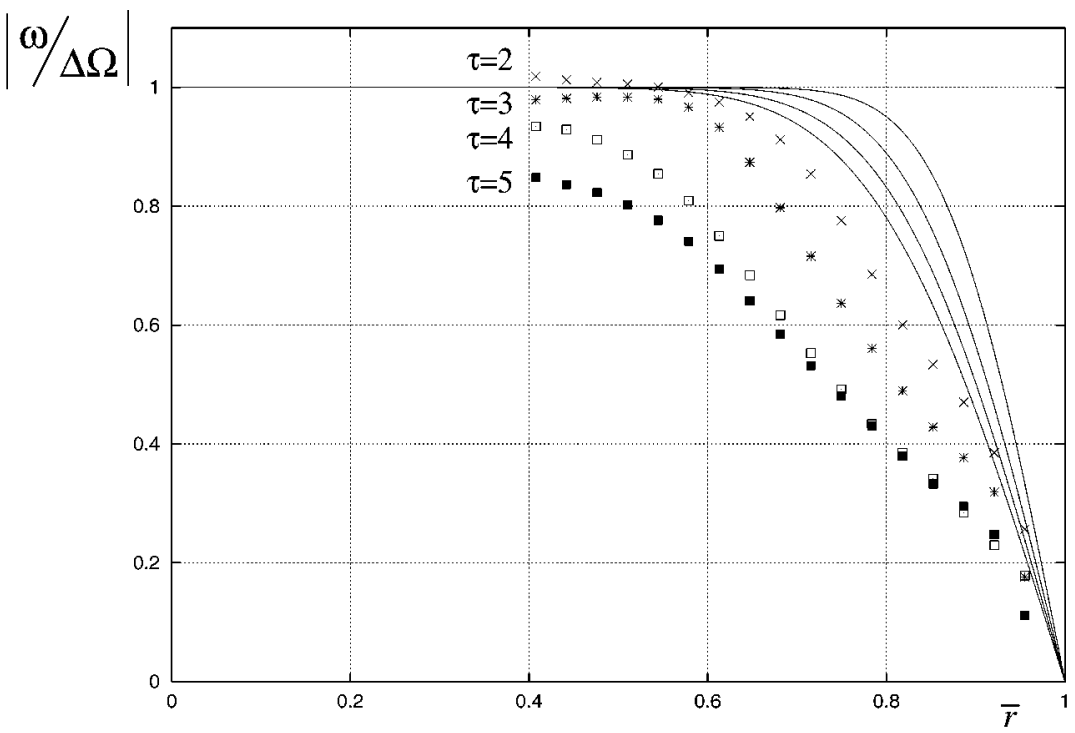

Figure 12 A comparison between experiment and boundary-layer predictions at $\tau=$ $2,3,4,5 ; \Omega=\left|\Omega_{i}-\Omega_{f}\right| ; \Omega_{i}=\pi / 5 s^{-1} ; \Omega_{f}=\pi / 10 s^{-1}$ (spin-down); and $N^{2}=2.5$ $s^{-2}$. Numerical results are obtained with $\sigma=700$, and the experimental results are an azimuthal average.
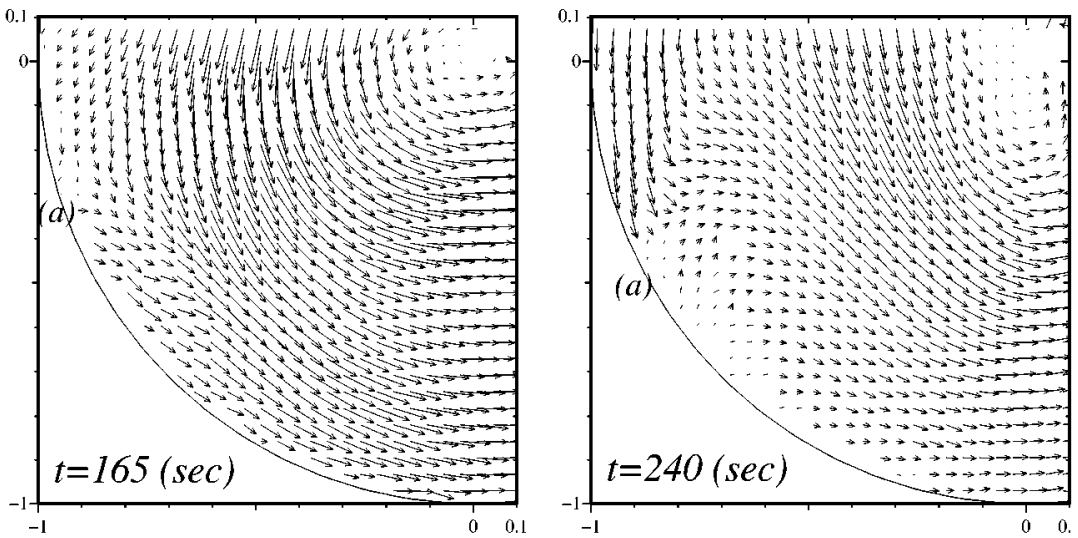

Figure 13 Two typical velocity fields for a spin-down experiment at $N^{2}=2.5 s^{-2}$, with $\Omega_{i}=\pi / 5 s^{-1}$ and $\Omega_{f}=\pi / 10 s^{-1}$. In each figure $(a)$ denotes a flow feature that develops in a continuous manner from that shown in the left-hand figure to that shown on the right. 


\section{ACKNOWLEDGMENTS}

The authors express their gratitude to Richard Hewitt and Peter Davies, who over the years have been superb coworkers. The EPSRC provided much of the funding for our work. We also thank Cambridge University Press for permission to reproduce a number of figures.

\section{Visit the Annual Reviews home page at www.AnnualReviews.org}

\section{LITERATURE CITED}

Allen JS. 1971. Some aspects of the initialvalue problem for the inviscid motion of a contained, rotating, weakly-stratified fluid. $J$. Fluid Mech. 46:1-23

Amberg G, Ungarish M. 1993. Spin-up from rest of a mixture: numerical simulation and asymptotic theory. J. Fluid Mech. 246:44364

Barcilon A, Lau J, Piacsek S, Warn-Varnas AC. 1975. Numerical experiments on stratified spin-up. Geophys. Fluid Dyn. 7:29-42

Beardsley RC, Saunders KD, Warn-Varnas AC, Harding JM. 1979. An experimental and numerical study of the secular spin-up of a thermally stratified rotating fluid. J. Fluid Mech. 93:161-84

Bennetts DA, Hocking LM. 1973. On nonlinear Ekman and Stewartson layers in a rotating fluid. Proc. R. Soc. London Ser. A 333:46989

Benton ER. 1973. Nonlinear hydrodynamic and hydromagnetic spin-up driven by EkmanHartmann boundary layers. J. Fluid Mech. 57:337-60

Benton ER, Clark A. 1974. Spin-up. Annu. Rev. Fluid Mech. 6:257-80

Benton ER, Loper DE. 1969. On the spinup of an electrically conducting fluid. Part 1. The unsteady hydromagnetic EkmanHartmann boundary-layer problem. J. Fluid Mech. 39:561-86

Berman AS, Bradford J, Lundgren TS. 1978. Two-fluid spin-up in a centrifuge. $J$. Fluid Mech. 84:411-31

Bodonyi RJ. 1978. On the unsteady similarity equations for the flow above a rotating disk in a rotating fluid. Q. J. Mech. Appl. Math. 31:461-72

Bodonyi RJ, Stewartson K. 1977. The unsteady laminar boundary layer on a rotating disk in a counter-rotating fluid. J. Fluid Mech. 79:669-88

Buzyna G, Veronis G. 1971. Spin-up of a stratified fluid: theory and experiment. J. Fluid Mech. 50:579-608

Cederlof U. 1988. Free-surface effects on spinup. J. Fluid Mech. 187:395-407

Chawla SS. 1976. Spin-up from a rotating disk. J. Fluid Mech. 78:609-19

Chawla SS. 1972. On hydromagnetic spin-up. J. Fluid Mech. 53:545-55

Choi S, Kim JW, Hyun JM. 1991. Experimental investigation of the flow with a free surface in an impulsively rotated cylinder. ASME J. Fluid Eng. 113:245-49

Choi S, Kim JW, Hyun JM. 1989. Transient free surface shape of an abruptly-rotated partially filled cylinder. ASME J. Fluid Eng. 111:43942

Clark A. 1973. The linear spin-up of a strongly stratified fluid of small Prandtl number. $J$. Fluid Mech. 60:561-80

Duck PW, Foster MR, Hewitt RE. 1997. On the boundary layer arising in the spin-up of a stratified fluid in a container with sloping walls. J. Fluid Mech. 335:233-59

Ekman VW. 1906. Beiträge zur Theorie der Meeresströmungen. Ann. Hydrograph. Marit. Meteorol. 2:1-50

Goller H, Ranov T. 1968. Unsteady rotating flow in a cylinder with a free surface. ASME J. Basic Eng. 90:445-54 
Greenspan HP. 1980. A note on the spin-up from rest of a stratified fluid. Geophys. Astrophys. Fluid Dyn. 15:1-5

Greenspan HP. 1968. The Theory of Rotating Fluids. Cambridge, UK: Cambridge Univ. Press. 327 pp.

Greenspan HP, Howard LN. 1963. On the time dependent motion of a rotating fluid. J. Fluid Mech. 17:385-404

Greenspan HP, Weinbaum S. 1965. On nonlinear spin-up of a rotating fluid. J. Math. Phys. 44:66-85

Hart J. 2000. A note on nonlinear corrections to the Ekman layer pumping velocity. Phys. Fluids 12:131-35

Henderson DM, Lopez JM, Stewart DL. 1996. Vortex evolution in non-axisymmetric impulsive spin-up from rest., J. Fluid Mech. 324:109-34

Hewitt RE, Davies PA, Duck PW, Foster MR. 1999. Spin-up of stratified rotating flows at large Schmidt number: experiment and theory. J. Fluid Mech. 389:169-207

Hewitt RE, Duck PW. 2000. Non-axisymmetric rotating-disk flows: nonlinear travelingwave states. J. Fluid Mech. 413:287-316

Hewitt RE, Duck PW, Foster MR. 1999. Steady boundary-layer solutions for a swirling stratified fluid in a rotating cone. J. Fluid Mech. 384:339-74

Hewitt RE, Duck PW, Foster MR, Davies PA. 1998. Nonlinear spin-up of a rotating stratified fluid: theory. ASME J. Fluids Eng. 120:662-66 (Proc. ASME/OED Int. Congr. Symp. 1997, 14:33-46)

Holton JR. 1965. The influence of viscous boundary layers on transient motions in a stratified rotating fluid, Parts 1 and 2. J. Atmos. Sci. 22:402-11

Holton JR, Stone PH. 1968. A note on the spinup of a stratified fluid. J. Fluid Mech. 33:12729

Homicz GF, Gerber N. 1986. Numerical model for fluid spin-up from rest in a partially filled cylinder. AIAA Pap. 86-1121, pp. 1-9

Homicz GF, Gerber N. 1987. Numerical model for fluid spin-up from rest in a partially filled cylinder. ASME J. Fluids Eng. 109:19597

Hyun JM. 1984. Flows within the Ekman layer during spin-up of a thermally stratified fluid. Geophys. Astrophys. Fluid Dyn. 29:6579

Hyun JM. 1983. Spin-up of a thermally stratified fluid in a cylinder with a conducting sidewall. Geophys. Astrophys. Fluid Dyn. 23:143-52

Hyun JM. 1982. Axisymmetric flows in spinup from rest of a stratified fluid in a cylinder. Geophys. Astrophys. Fluid Dyn. 23:127-41 Hyun JM, Fowlis WW, Warn-Varnas A. 1982. Numerical solutions for the spin-up of a stratified fluid. J. Fluid Mech. 117:71-90

Hyun JM, Leslie F, Fowlis WW, Warn-Varnas A. 1983. Numerical solutions for spin-up from rest in a cylinder. J. Fluid Mech. 127: 263-81

Hyun JM, Park JS. 1992. Spin-up from rest of a compressible fluid in a rapidly rotating cylinder. J. Fluid Mech. 237:413-34

Ibrani S, Dwyer H. 1987. Flow interactions during axisymmetric spin up. AIAA J. 25:130511

Kerswell RR. 1999. Secondary instabilities in rapidly rotating fluids: inertial wave breakdown. J. Fluid Mech. 382:283-306

Kitchens CW. 1980. Navier-Stokes solutions for spin-up in a filled cylinder. AIAA $J$. 18:929-34

Lee SM. 1975. An investigation of stratified spin-up using a rotating laser-Doppler velocimeter. MS thesis. Fla. State Univ., Tallahassee.

Lindblad IAA, Bark FH, Zahrai S. 1994. Spinup of a rapidly rotating heavy gas in a thermally insulated annulus. J. Fluid Mech. 274:383-404

Loper DE. 1971. Hydromagnetic spin-up of a fluid confined by two flat electrically conducting boundaries. J. Fluid Mech. 50:60923

Loper DE, Benton ER. 1970. On the spin-up of an electrically conducting fluid. Part 2. Hydromagnetic spin-up between infinite flat 
insulating plates. J. Fluid Mech. 43:785-99

Lopez JM. 1996. Flow between a stationary and a rotating disk shrouded by a co-rotating cylinder. Phys. Fluids 8:2605-13

Lopez JM, Weidman PD. 1996. Stability of stationary endwall boundary layers during spindown. J. Fluid Mech. 326:373-98

MacCready P, Rhines PB. 1991. Buoyant inhibition of Ekman transport on a slope and its effect on stratified spin-up. J. Fluid Mech. 223:631-61

Mathis DM, Neitzel GP. 1985. Experiments on impulsive spin-down to rest. Phys. Fluids 28:449-54

Maynes D, Klewicki J, McMurtry P. 1999. Spinup in a tank induced by a rotating bluff body. J. Fluid Mech. 388:49-68

McDonald BE, Dicke RH. 1967. Solar oblateness and fluid spin-down. Science 158:156264

Modisette JL, Novotny JE. 1969. Buoyancy and solar spin-down. Science 166:872-74

Neitzel P, Davis SH. 1981. Centrifugal instabilities during spin-down to rest in finite cylinders. Numerical experiments. J. Fluid Mech. 102:329-52

O’Donnell J, Linden PF. 1991. Free-surface effects on the spin-up of fluid in a rotating cylinder. J. Fluid Mech. 232:439-53

Pedlosky J. 1967. The spin up of a stratified fluid. J. Fluid Mech. 28:463-79

Pettersson H. 1931. Über das sogenannte Schaumrandphänomen. Göteb. Kungl. Vetensk. Vitterh.-Samh. Handl. 2:1-21

Rogers MH, Lance GN. 1960. The rotationally symmetric flow of a viscous fluid in the presence of an infinite rotating disk. J. Fluid Mech. 7:617-631

Sakurai T. 1969. Spin down problem of rotating stratified fluid in thermally insulated circular cylinders. J. Fluid Mech. 37:689-99

Sakurai T, Clark A, Clark PA. 1971. Spin-down of a Boussinesq fluid of small Prandtl number in a circular cylinder. J. Fluid Mech. 49:75373

Saunders KD, Beardsley RC. 1975. An experimental study of the spin-up of a thermally stratified rotating flow. J. Geophys. Fluid Dyn. 7:1-28

Siegmann WL. 1971. The spin down of rotating stratified fluids. J. Fluid Mech. 47:689-711

Spence GSM, Foster MR, Davies PA. 1992. The transient response of a contained rotating stratified fluid to impulsively started surface forcing. J. Fluid Mech. 243:33-50

Stewartson K. 1957. On almost rigid rotations. J. Fluid Mech. 3:17-26

Stewartson K, Simpson CJ, Bodonyi RJ. 1982. The unsteady boundary layer on a rotating disk in a counter-rotating fluid. Part 2. J. Fluid Mech. 121:507-15

St-Maurice J-P, Veronis G. 1975. A multiscaling analysis of the spin-up problem. $J$. Fluid Mech. 68:417-45

Suh YK. 1994. Numerical study on twodimensional spin-up in a rectangle. Phys. Fluids 6:2333-44

Thorpe SA. 1987. Current and temperature variability on the ontinental slope. Philos. Trans. R. Soc. London Ser. A A323:471-517

Ungarish M. 1997. The spin-up of liquid metal driven by a rotating magnetic field. J. Fluid Mech. 347:105-18

van de Konijnenberg JA. 1995. Spin-up in non-axisymmetric containers. $\mathrm{PhD}$ diss. Eindhoven Univ. Technol. Eindhoven, The Netherlands. $156 \mathrm{pp}$.

van de Konijnenberg JA, Andersson HI, Billdal JT, van Heijst GJF. 1994. Spin-up in a rectangular container with low angular velocity. Phys. Fluids 6:1168-76

van de Konijnenberg JA, van Heijst GJF. 1997. Free-surface effects on spin-up in a rectangular tank. J. Fluid Mech. 334:189-210

van de Konijnenberg JA, Wessels TL, van Heijst GJF. 1996. Spin-up in a circular tank with a radial barrier. Phys. Fluids 8:204859

van Heijst GJF. 1989. Spin-up phenomena in non-axisymmetric containers. J. Fluid Mech. 206:171-91

van Heijst GJF, Davies PA, Davis RG. 1990. Spin-up in a rectangular container. Phys. Fluids A 2:150-59 
Venezian G. 1970. Nonlinear spin-up. Top. Ocean Eng. 2:87-96

von Kármán T. 1921. Uber laminare und turbulente Reiburg. Z. Angew. Math. Mech. 1:23352

Walin G. 1969. Some aspects of time-dependent motion of a stratified rotating fluid. J. Fluid Mech. 36:289-307

Warn-Varnas A, Fowlis WW, Piacsek S, Lee SM. 1978. Numerical solutions and laserDoppler measurements of spin-up. J. Fluid Mech. 85:609-39

Watkins WB, Hussey RG. 1977. Spin-up from rest in a cylinder. Phys. Fluids 20:1596-604

Watkins WB, Hussey RG. 1973. Spin-up from rest: limitations of the Wedemeyer model. Phys. Fluids 16:1530-31
Wedemeyer EH. 1964. The unsteady flow within a spinning cylinder. J. Fluid Mech. 20:383-99

Weidman PD. 1976a. On the spin-up and spindown of a rotating fluid. Part 1. Extending the Wedemeyer model. J. Fluid Mech. 77:685708

Weidman PD. 1976b. On the spin-up and spindown of a rotating fluid. Part 2. Measurements and stability. J. Fluid Mech. 77:70935

Zandbergen PJ, Dijkstra D. 1987. Von Kármán swirling flows. Annu. Rev. Fluid Mech. 19:465-91

Zeipel HV. 1924. The radiation equilibrium of a rotating system of gaseous masses. MNRAS $84: 665,684,702$ 
赵 Annual Review of Fluid Mechanics

Volume 33, 2001

\section{CONTENTS}

James Lighthill and His Contributions to Fluid Mechanics, TJ Pedley 1

Steady Streaming, N Riley 43

On the Fluid Mechanics of Fires, Sheldon R Tieszen 67

Experiments on Thermocapillary Instabilities, Michael F Schatz and G Paul Neitzel

Robert Legendre and Henri Werlé: Toward the Elucidation of Three-Dimensional Separation, Jean M Délery

Surface Pressure Measurements Using Luminescent Coatings, James H Bell, Edward T Schairer, Lawrence A Hand, and Rabindra D Mehta

Rossby Wave Hydraulics, ER Johnson and SR Clarke 207

Spin-Up of Homogeneous and Stratified Fluids, PW Duck and MR Foster 231

Extrusion Instabilities and Wall Slip, Morton M Denn 265

Turbulent Relative Dispersion, Brian Sawford $\quad 289$

Early Work on Fluid Mechanics in the IC Engine, John L Lumley 319

Mechanics of Coastal Forms, Paolo Blondeaux 339

Aerodynamics of High-Speed Trains, Joseph A Schetz 371

Junction Flows, Roger L Simpson 415

Modeling of Fluid-Structure Interaction, Earl H Dowell and Kenneth C Hall

Compression System Stability and Active Control, JD Paduano, EM Greitzer, and AH Epstein

Spilling Breakers, JH Duncan

Shelterbelts and Windbreaks: Mathematical Modeling and Computer Simulations of Turbulent Flows, Hao Wang, Eugene S Takle, and Jinmei Shen

Drag Due to Lift: Concepts for Prediction and Reduction, Ilan Kroo

Inertial Effects in Suspension and Porous-Media Flows, Donald L Koch and Reghan J Hill 


\section{INDEXES}

Subject Index

Cumulative Index of Contributing Authors, Volumes 1-33

Cumulative Index of Chapter Titles, Volumes 1-33 